\title{
Modular and Distinct Plexin-A4/FARP2/Rac1 Signaling Controls Dendrite Morphogenesis
}

\author{
Victor Danelon, ${ }^{1 \star}$ Ron Goldner, ${ }^{2 \star}$ Edward Martinez, ${ }^{1}$ Irena Gokhman, ${ }^{2}$ Kimberly Wang, ${ }^{1}$ Avraham Yaron, ${ }^{2}$ \\ and ${ }^{(}$Tracy S. Tran ${ }^{1}$ \\ ${ }^{1}$ Department of Biological Sciences, Rutgers University, Newark, New Jersey 07102, and ${ }^{2}$ Department of Biomolecular Sciences, Weizmann Institute \\ of Science, Rehovot, 76100, Israel
}

Diverse neuronal populations with distinct cellular morphologies coordinate the complex function of the nervous system. Establishment of distinct neuronal morphologies critically depends on signaling pathways that control axonal and dendritic development. The Sema3A-Nrp1/PlxnA4 signaling pathway promotes cortical neuron basal dendrite arborization but also repels axons. However, the downstream signaling components underlying these disparate functions of Sema3A signaling are unclear. Using the novel PlxnA4 ${ }^{K R K-A A A}$ knock-in male and female mice, generated by CRISPR/cas9, we show here that the KRK motif in the PlxnA4 cytoplasmic domain is required for Sema3A-mediated cortical neuron dendritic elaboration but is dispensable for inhibitory axon guidance. The RhoGEF FARP2, which binds to the KRK motif, shows identical functional specificity as the KRK motif in the PlxnA4 receptor. We find that Sema3A activates the small GTPase Racl, and that Rac1 activity is required for dendrite elaboration but not axon growth cone collapse. This work identifies a novel Sema3A-Nrp1/PlxnA4/FARP2/Rac1 signaling pathway that specifically controls dendritic morphogenesis but is dispensable for repulsive guidance events. Overall, our results demonstrate that the divergent signaling output from multifunctional receptor complexes critically depends on distinct signaling motifs, highlighting the modular nature of guidance cue receptors and its potential to regulate diverse cellular responses.

Key words: axonal repulsion; dendritic branching; guidance receptors; neural development; Semaphorin signaling

Significance Statement

The proper formation of axonal and dendritic morphologies is crucial for the precise wiring of the nervous system that ultimately leads to the generation of complex functions in an organism. The Semaphorin3A-Neuropilin1/Plexin-A4 signaling pathway has been shown to have multiple key roles in neurodevelopment, from axon repulsion to dendrite elaboration. This study demonstrates that three specific amino acids, the KRK motif within the Plexin-A4 receptor cytoplasmic domain, are required to coordinate the downstream signaling molecules to promote Sema3A-mediated cortical neuron dendritic elaboration, but not inhibitory axon guidance. Our results unravel a novel Semaphorin3A-Plexin-A4 downstream signaling pathway and shed light on how the disparate functions of axon guidance and dendritic morphogenesis are accomplished by the same extracellular ligand in vivo.

Received Nov. 17, 2019; revised Apr. 29, 2020; accepted May 26, 2020.

Author contributions: V.D., R.G., E.M., I.G., and K.W. performed research; V.D., R.G., A.Y., and T.S.T. analyzed data; V.D. and R.G. wrote the first draft of the paper; V.D., R.G., A.Y., and T.S.T. edited the paper; A.Y. and T.S.T. designed research; A.Y. and T.S.T. wrote the paper.

The authors declare no competing financial interests.

*V.D. and R.G. contributed equally to this work.

This work was support by National Science Foundation Integrative Organismal Systems BOI Grant 1556968 and BSF Grant 2013052 to T.S.T. and A.Y. Research in the laboratory of T.S.T. was also supported by the New Jersey Governor's Council for Medical Research and Treatment of Autism Grant CAUT17BSP022, the New Jersey Commission on Spinal Cord Research Grant CSCR16IRG013, and Rutgers University Newark Chancellor's Initiative for Multidisciplinary Research Teams Award. Research in the laboratory of A.Y. was supported by the Jeanne and Joseph Nissim Center for Life Sciences Research at the Weizmann Institute of Science and the Nella and Leon Benoziyo Center for Neurologic Diseases. A.Y. is an incumbent of the Jack and Simon Djanogly Professorial Chair in Biochemistry. We thank Alex Kolodkin and Oren Schuldiner for providing critical feedback to this work; and Adefemi Baderinwa and Michelle Davis for excellent technical support with mouse genotyping.

Correspondence should be addressed to Tracy S. Tran at tstran@rutgers.edu or Avraham Yaron at avraham.yaron@weizmann.ac.il.

https://doi.org/10.1523/JNEUROSCI.2730-19.2020

Copyright $\odot 2020$ the authors

\section{Introduction}

The development of precise neuronal connections critically depends on proper extension of axons and elaboration of dendrites. Studies of neurite outgrowth and axon guidance are well established (Barnes and Polleux, 2009; Kolodkin and TessierLavigne, 2011; Stoeckli, 2018); and while numerous studies were conducted in invertebrates (Jan and Jan, 2010), much less is known about the signaling mechanisms controlling dendritic morphogenesis in the mammalian nervous system. Interestingly, molecular cues initially described as axonal guidance cues also control later events in dendrite development, suggesting that these cues are multifunctional (Whitford et al., 2002; Yaron et al., 2005; Tran et al., 2009; Smith et al., 2012; Mlechkovich et al., 2014; Nagel et al., 2015; Anzo et al., 2017).

The Semaphorin family of multifunctional cues has been mainly studied in the context of axon guidance (Jin and Strittmatter, 1997; Zanata et al., 2002; Huber et al., 2003; 
Toyofuku et al., 2005; Tong et al., 2007; Tran et al., 2007). Semaphorins can repel or attract axons by engaging different coreceptors (Castellani et al., 2000; Chauvet et al., 2007) or interacting with molecules in the local environment (Kantor et al., 2004). Interestingly, the Class 3 secreted Semaphorin-3A (Sema3A) can repel axons and promote dendrite branching in different neuronal populations both in vitro and in vivo (Gu et al., 2003; Fenstermaker et al., 2004; Yaron et al., 2005; Tran et al., 2009; Mlechkovich et al., 2014). However, the intracellular events orchestrating these divergent outputs, elicited by the same Sema3A cue, are largely elusive.

Sema3A signaling requires the binding to its obligate partner Neuropilin-1 (Nrp1) and formation of a complex with one of the Type-A Plexins (Plxns) (Tran et al., 2007). However, little was known about the specific Plxn signaling receptor involved in dendrite development until we showed the reduced dendritic complexity of PlxnA4 KO deep cortical neurons in vivo (Tran et al., 2009) that phenocopied those observed in the Nrp1 $1^{\text {sema- }}$ mutants (Gu et al., 2003). We demonstrated in vitro that the ability of Sema3A to mediate growth cone collapse in DRG neurons versus dendritic arborization of cortical neurons is embedded in a modular nature of the PlxnA4 receptor, within its distinct cytoplasmic domains (Mlechkovich et al., 2014). We showed that the KRK motif in PlxnA4 can associate with the RhoGEF FARP2 and is required for dendrite elaboration in vitro. However, the downstream signaling elements controlling dendrite elaboration versus axon guidance remained elusive.

Regulators of small GTPases (GEFs and GAPs) may represent signaling-specificity elements for divergent Semaphorin functions. The RacGAP $\beta 2$-Chimaerin is required for Sema3Finduced axonal pruning but not repulsion (Riccomagno et al., 2012). The PlxnA2 GAP activity is required for proper distribution of dentate gyrus granule cells, but not for mossy fiber-CA3 targeting in the developing hippocampus (Zhao et al., 2018). These studies, along with our work, suggest that Nrp/PlxnA complexes can recruit different GEFs and GAPs through distinct motifs, or the GAP domain itself, within the receptor to induce distinct cellular responses. Thus, we hypothesize that the KRK motif in PlxnA4 could serve as a recruitment site for specific GEFs, such as FARP2, to selectively confer Sema3A-dependent dendrite morphogenesis in cortical neurons in vivo.

Here, we generated a mouse line harboring an AAA substitution of the basic KRK motif in the PlxnA4 intracellular domain. Using this mouse line, we show that this conserved motif is required for Sema3A-induced dendritic elaboration in cortical neurons, but not for axonal repulsion or growth inhibition of several axonal tracts in the PNS and CNS. We demonstrate that cortical neuron dendrite development mediated by PlxnA4 signaling requires FARP2 in vivo. Furthermore, we show that Sema3A induces Rac1 activation in a FARP2-dependent manner in cortical neurons, and that this activity is selectively required for dendritic arborization, but not for growth cone collapse. Our work uncovers a distinct pathway for dendritic elaboration, specifically engaging the KRK motif of the multifunctional PlxnA4 receptor and its downstream signaling effectors.

\section{Materials and Methods}

\section{Mouse strains}

Mice harboring a point mutation in which the intracellular KRK (lysinearginine-lysine) motif of Plexin-A4 was replaced by a triplet of alanines $\left(P l x n A 4^{K R K>A A A}\right)$ were generated by CRISPR/Cas9-based homologous recombination of a $164 \mathrm{bp}$ ssDNA oligonucleotide containing the KRK $\left(5^{\prime}\right.$-AAACGAAAA-3') to AAA (5'-GCAGCAGCA-3') mutation. A guide RNA (5'-CGCCATTGTCAGCATCGCGG-3') was designed to target exon 20 of PlxnA4 with minimal off-target effects. The ssDNA oligonucleotide had 76-79 bp homology arms around the KRK $>$ AAA point mutation, with disruption of the TGG PAM sequence to TAG. In vitro transcribed Cas9 RNA $(100 \mathrm{ng} / \mu \mathrm{l})$, the sgRNA $(50 \mathrm{ng} / \mu \mathrm{l})$, together with the ssDNA $(200 \mathrm{ng} / \mu \mathrm{l})$, were injected into one cell-fertilized embryos isolated from superovulated CB6F1 hybrid mice (F1 of BALB/ cAnNHsd inbred female $\times$ C57BL/6NHsd inbred male) mated with CB6F1 males from Harlan Biotech Israel. Injected embryos were transferred into the oviducts of pseudopregnant ICR females. Screening of tail tip DNAs was conducted by PCR genotyping and confirmed by sequencing. Heterozygous males were backcrossed with females of CD1 (ICR) genetic background.

For generation of the Farp2 KO mouse line, two CRISPR sgRNAs were designed flanking a 18,167 bp sequence in the Farp2 locus on Chr1 (GRCm38-mm10), including the promoter, the first utr exon and the second exon (with ATG). One sgRNA (5'-TCTTAAGGACTTATTG CCAA-3', chromosome 1: 93511211-93511211) was targeted to a region upstream of the Farp2 gene promoter, and a second sgRNA (5' GTGAGGGCCTCATTCCGAAA-3', chromosome 1: 93529378-93529397) to intron 2. The sgRNAs were designed using several CRISPR designing tools, and optimized for the best guides, including the following: the MIT CRISPR design tool (Hsu et al., 2013) and sgRNA Designer, Rule Sets 1 and 2 (Doench et al., 2014, 2016), in both the original sites and later in the Benchling implementations (www.benchling.com), SSC (Xu et al., 2015), and sgRNA scorer (Chari et al., 2015), in their websites. In vitro transcribed Cas9 RNA (100 ng/ $\mu \mathrm{l})$ and the sgRNAs $(50 \mathrm{ng} / \mu \mathrm{l})$ were injected into one cell-fertilized embryos isolated from superovulated $\mathrm{CB} 6 \mathrm{~F} 1$ hybrid mice ( $\mathrm{F} 1$ of $\mathrm{BALB} / \mathrm{cAnNHsd}$ inbred female $\times \mathrm{C} 57 \mathrm{BL} / 6 \mathrm{NHsd}$ inbred male) mated with $\mathrm{CB} 6 \mathrm{~F} 1$ males from Harlan Biotech Israel. Injected embryos were transferred into the oviducts of pseudopregnant ICR females. Screening of tail tip DNAs was conducted by PCR genotyping and RT-PCR (see Fig. 1). Heterozygous males were backcrossed with females of CD1(ICR) genetic background.

To investigate the role of FARP2 in mediating Sema3A cortical dendritic arborization, we used Farp $2 \mathrm{KO}$ mice generated by Takegahara et al. (2010). The following oligonucleotide primers were used to identify the rearranged Farp2 locus: primer 1 (5-ATCAAACTCCACCCTGA GGTCCATG-3), primer 2 (5-TTTGTAAACTGCAGCGTTTCTCTTC3), and primer 3 (5-CTTCTGAGGGGATCGGCAAT-3).

Mice harboring the Thyl-EGFP (M line) transgene were obtained at The Jackson Laboratory (stock \#007788 Tg-Thyl-EGFP-MJrs/J) and maintained by breeding with female C57BL/6J WT mice. PlxnA4 KO mice were previously described (Yaron et al., 2005). Embryonic day (E) 0.5 was considered to be the day a vaginal plug was found. Newborn pups were weaned at postnatal day (P) 21 and housed under a $12 \mathrm{~h} \mathrm{light/}$ $12 \mathrm{~h}$ dark cycle at constant temperature with food and water ad libitum. All animal work in this study has been approved by the Institutional Animal Care and Use Committees Rutgers University Newark and the Weizmann Institute of Science.

\section{Primary neuron cultures}

For the growth cone collapse assay, DRGs from E13.5 embryos were dissected and plated as explants in chambers coated with $10 \mu \mathrm{g} / \mathrm{ml}$ poly-D-lysine (Sigma Millipore, catalog \#P6407) and $10 \mu \mathrm{g} / \mathrm{ml}$ laminin (Sigma Millipore, catalog \#L2020). Growing media were as follows: Neurobasal-A (Thermo Fisher Scientific, catalog \#10888-022) supplemented with 2\% B-27 (Invitrogen, catalog \#17504044), 1\% penicillin-streptomycin (Biological Industries, catalog \#03-031-1B), 1\% L-glutamine (Biological Industries, catalog \#03-020-1B), and $25 \mathrm{ng} / \mathrm{ml}$ NGF (Alomone Labs, catalog \#AN-240). After 48 h, explants were treated with $0.1,0.5$, or $1 \mathrm{~nm}$ AP-Sema3A for $30 \mathrm{~min}$, then fixed with $4 \%$ PFA $+15 \%$ sucrose solution, and stained with phalloidin-rhodamine (Sigma Millipore, catalog \#P1951, 1:250) for visualization of F-actin filopodia. Growth cones with no or few filopodia were considered as collapsed. Percentage of collapsed growth cones was calculated for each treatment group and control.

For the collagen axonal repulsion assay, DRGs from E13.5 embryos were dissected and placed in droplets of $2 \mathrm{mg} / \mathrm{ml}$ collagen (Roche 
A

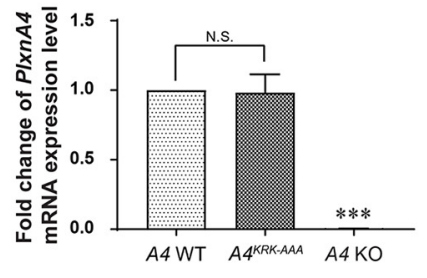

D

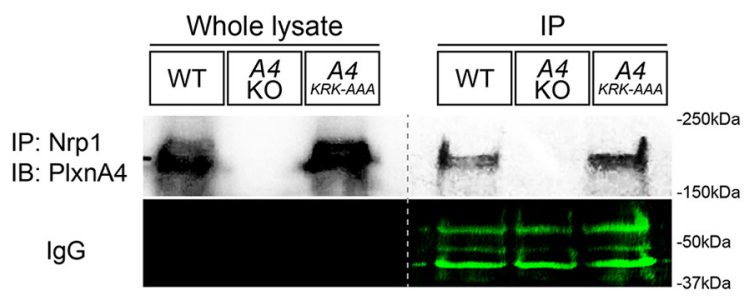

E

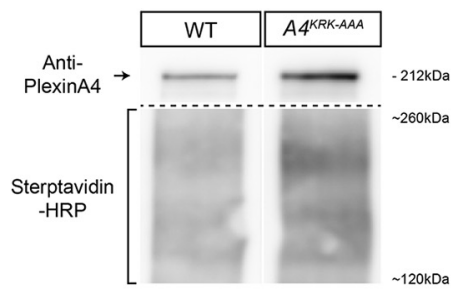

F

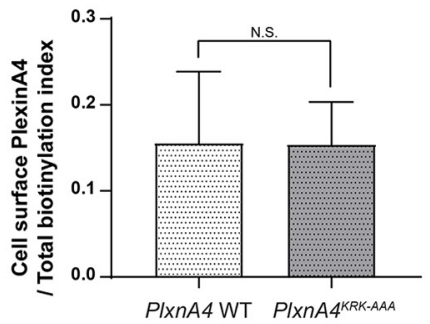

B

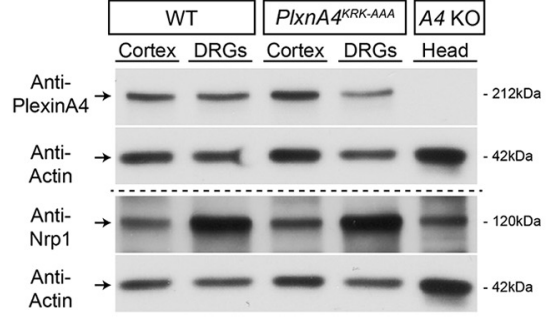

$37 \mathrm{kDa}$

H
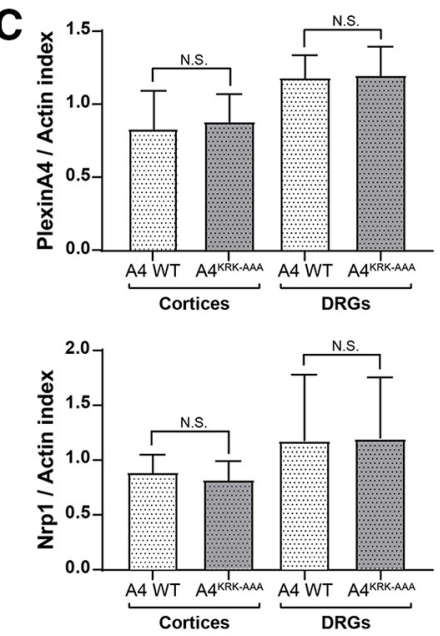

G

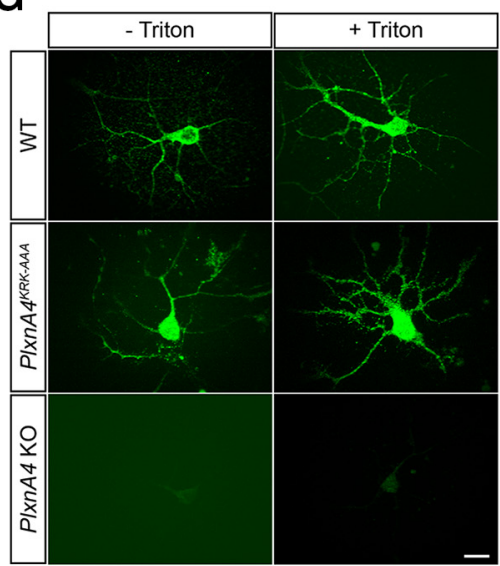

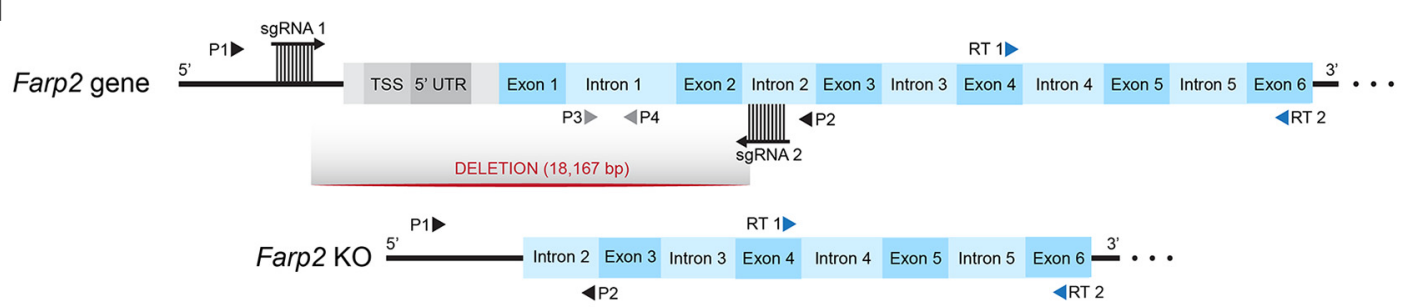

I

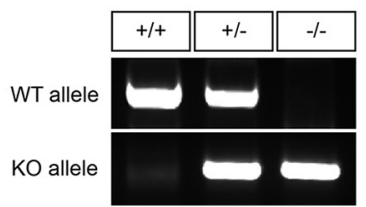

J

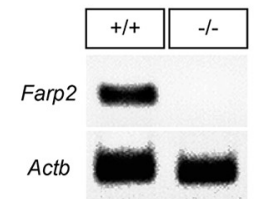

Figure 1. Generation of the PlxnA4 ${ }^{K R K-A A A}$ mutant mouse line and the Farp2 KO mouse line. A, qRT-PCR analysis revealed unchanged PlxnA4 mRNA transcript levels in E13.5 PlxnA4 $4^{K R K-A A A}$ heads compared with WT littermate controls. PlxnA4 KO heads were processed as well as a negative control. Quantification was performed using a relative quantification analysis $\left(\Delta \Delta C_{t}\right)$ and presented as mean \pm SEM. ${ }^{* * *} p<0.001$ (Student's $t$ test). B, C, Western blot analysis of E13.5 DRGs and cortices extracted from PlxnA4 ${ }^{\text {KRK-AAA }}$ and WT littermate controls revealed similar Plexin-A4 and Nrp1 protein levels in both genotypes. Heads of E13.5 PlxnA4 KO were used as a negative control. Data are mean \pm SEM. Statistical analysis: Student's $t$ test. D, PlxnA4 ${ }^{K R K-A A A}$, WT littermates, and PlxnA4 K0 cortices were subjected to anti-Nrp1 immunoprecipitation and probed using a Plexin-A4 antibody. No change in Nrp1-Plexin-A4 association was detected in the PIXnA4 ${ }^{\text {KRK-AAA }}$ mutant compared with WT. E, F, 7 DIV cortical neurons from E13.5 PIxnA4 ${ }^{\text {KRK-AAA }}$ and WT littermate embryos were subjected to a cell-surface biotinylation assay, followed by Western blot probing for Plexin-A4. The total mass of biotinylated proteins in the range of $\sim 120-260 \mathrm{kDa}$, detected and quantified using streptavidin-HRP, was used as loading control. Data are mean \pm SEM; N.S., Non Significant. G, Primary cortical neurons of PIxnA4 ${ }^{K K K-A A A}$, WT littermates, and PlxnA4 KO embryos were immunostained in culture using a Plexin-A4 antibody. Plexin-A4 protein levels and its spatial pattern in the PlxnA4 ${ }^{K K K \text {-AAA }}$ mutant were comparable with the WT, whereas virtually no Plexin-A4 was detected in the negative control KO. Scale bar, $25 \mu \mathrm{m}$. $\boldsymbol{H}$, Schematic representation of the Farp2 locus on chromosome 1 (GRCm38-mm10) and the CRISPR-Cas9 K0 design. Two sgRNAs flanking an 18,167 bp sequence were used: sgRNA 1 targeted a region upstream of the Farp2 gene promoter, and sgRNA 2 targeted a sequence in intron 2. P1 and P2 (black triangles) represent primers used for detection of the K0 allele in a PCR analysis of genomic DNA, targeting sequences outside of the deletion area (upstream of sgRNA1 and downstream of sgRNA2, respectively). Similarly, P3 and P4 (gray triangles) represent primers used for detection of the WT allele and were targeted at intron 1. RT 1 and 2 (blue triangles) represent primers used for reverse transcription analysis of Farp2 gene expression levels; RT 1 targeted a sequence comprised of the $3^{\prime}$ of exon 4 and 5' of exon 5, and RT 2 targeted exon 6. I, PCR analysis of tail genomic DNA obtained from three E13.5 embryos of a Farp2 Het X 
Diagnostic, catalog \#11179179001) together with COS1 aggregates transfected with either secreted Myc-Sema3A or control PAY1-GFP. Cocultures were grown for $48 \mathrm{~h}$ in the aforementioned Neurobasal-A growth medium $+12.5 \mathrm{ng} / \mathrm{ml} \mathrm{NGF}$, then fixed with $4 \% \mathrm{PFA}$, and stained with anti-tubulin $\beta$ III antibody (R\&D systems, Tuj1 clone, 1:1000). Extent of axonal repulsion was measured by the ratio between the lengths of the proximal axons (i.e., axons that are close to the COS1 aggregate) to the length of the distal axons.

Cortical neurons were obtained from E13.5 WT, or from PlxnA4 $\mathrm{KO}, P l \times n A 4^{K R K-A A A}$ and Farp2 KO mice as previously described (Mlechkovich et al., 2014). When the experiments required, dissociated neurons were transfected with Farp2-HA-tagged (Mlechkovich et al., 2014) using Amaxa Mouse Neuron Nucleofector Kit catalog \#VPG1001. The neurons were plated onto glass coverslips (coated with polyD-lysine, $0.1 \mathrm{mg} / \mathrm{ml}$, catalog \#P6407) in 12-well plates (TPP 92412) and cultured with Neurobasal growth medium supplemented with 2\% B-27 (catalog \#17 504-044), 1\% penicillin/streptomycin (catalog \#15140122), and $1 \%$ Glutamax (catalog \#35050) for 4-5 d.

\section{Production of AP-Sema3A}

Alkaline phosphatase (AP)-tagged Sema3A was produced in HEK293T cells as previously described (Mlechkovich et al., 2014). Briefly, HEK293T cells (ATCC catalog \#CRL-3216, RRID:CVCL_0063) were grown in DMEM supplemented with 10\% FBS (VWR, catalog \#97068$085)$ and $1 \%$ penicillin-streptomycin. The cells were transfected using BioT (catalog \#B01-01) with an AP-Sema3A expression vector (Huber et al., 2005). The conditioned medium was concentrated using Amicon ultra-15 UFC 910024 (Sigma Millipore). AP-Sema3A concentration was determined by AP activity assay (using AP substrate buffer para-nitrophenyl phosphate, Sigma Millipore) (Mlechkovich et al., 2014).

\section{Rac1 activity assay}

Racl activation was measured by using a Racl activation assay kit (Rac1 Pull-down Activation Assay Biochem Kit bead pulldown format, Cytoskeleton, catalog \#BK035), following the manufacturer's protocol, with minor modifications. Briefly, fresh lysates of primary cortical neurons at DIV 5 incubated either with AP ( $5 \mathrm{nM})$ or Sema3A-AP ( $5 \mathrm{nM})$ for 30 min were incubated with the glutathione S-transferase (GST)-fused p21-binding domain of PAK1 (GST-PAK1-PBD) at $4^{\circ} \mathrm{C}$ for $1 \mathrm{~h}$ with gentle shaking, followed by pelleting of the PAK1-PBD beads by centrifugation at 3-5000 $\times g$ at $4^{\circ} \mathrm{C}$ for $1 \mathrm{~min}$. After one wash with wash buffer, the beads were eluted in reduced $2 \times$ SDS sample buffer (Laemmli buffer) and analyzed by Western blot. The Rac1 antibody used was antiRac1 (Abcam, catalog \#ab33186), concentration $0.6 \mu \mathrm{g} / \mathrm{ml}$.

\section{Rac inhibitor EHT1864}

For cortical neurons, EHT1864 (Tocris Bioscience, catalog \#3872, batch \#1J/197009), inhibitor of Rac family GTPases (Shutes et al., 2007; Onesto et al., 2008), was prepared following manufacturer guidelines. Cortical neurons were incubated with EHT1864 at different concentrations (2.5, 5 , and $10 \mu \mathrm{M}$ ) (Gaitanos et al., 2016) for $2 \mathrm{~h}$ at $37^{\circ} \mathrm{C}$ before treatment and also added with either $5 \mathrm{~nm}$ of AP-Sema3A or AP alone treatment. DRG explants were incubated with the specified EHT1864 concentration for $30 \mathrm{~min}$ at $37^{\circ} \mathrm{C}$ before Sema3 A bath application. The same volume of $\mathrm{H}_{2} \mathrm{O}$ was added to the no inhibitor treatment control cells.

\section{Rac1 siRNA}

The siRNA was purchased from Dharmacon. Racl siRNA: catalog \#M041170-01-0005, siGenome Mouse Racl (19353) siRNA-SMART pol, $5 \mathrm{nmol}$. Target sequences: D-041170-01, GGACGAAGCUUGAUCUU AG; D-041170-02, AGACGGAGCUGUUGGUAAA; D-041170-03, GAU

Het cross, using primers 1-4 (P1-P4). Lengths of the WT and K0 alleles are 551 and 271 bp, respectively. + /+, WT; +/-, Farp2 heterozygous; - /-, Farp2 K0. J, Reverse transcription analysis of WT $(+/+)$ and KO $(-/-)$ CDNA using either Farp2 primers RT 1-2 (top), or Actin $\beta$ (Actb) primers as internal control (bottom). $\boldsymbol{G}, \boldsymbol{H}$, Successful K0 of the Farp2 gene and its transcripts.
CGGUGCUGUCAAAUAC; and D-041170-04, GCAAAGUGGUAUCC UGAAG.

Scrambled siRNA: catalog \#D-001206-13-05, siGENOME NonTargeting siRNA Pool \#1, $5 \mathrm{nmol}$. Target sequences: UAGCGACUA AACACAUCAA, UAAGGCUAUGAAGAGAUAC, AUGUAUUGGCC UGUAUUAG, and AUGAACGUGAAUUGCUCAA. The Rac1 siRNA or the Scramble was transfected to cortical neurons using Amaxa Mouse Neuron Nucleofector Kit catalog \#VPG-1001.

\section{Immunohistochemistry}

After 4-5 DIV, cortical neurons were treated with $5 \mathrm{~nm}$ AP-Sema3A or $\mathrm{AP}$ alone for $24 \mathrm{~h}$, fixed in $4 \% \mathrm{PFA}$ at $4^{\circ} \mathrm{C}$ for $15 \mathrm{~min}$, and processed for immunocytochemistry, as previously described (Mlechkovich et al., 2014). Primary antibodies used were as follows: monoclonal rabbit antiMAP2 (1:1000, Cell Signaling Technology, 4542S) and monoclonal mouse anti-HA (1:500, Santa Cruz Biotechnology, F7 sc-7392). Secondary fluorescently conjugated antibodies were AlexaFluor-488 donkey anti-mouse IgG (1:500, Jackson ImmunoResearch Laboratories) and Cy3 donkey anti-rabbit (1:1000, Jackson ImmunoResearch Laboratories). Coverslips were mounted on microscope slides using Mowiol (Sigma Millipore, catalog \#81381)/10\% p-phenylenediamine (catalog \#78460).

For cell surface expression of PlxnA4 and PlxnA4 ${ }^{\text {KRK-AAA }}$ protein, nonpermeabilized primary cortical neurons were immunostained using 1:1000 rabbit monoclonal Plexin-A4 (C5D1).

For whole-mount Neurofilament staining, embryos at E12.5 were collected into $1 \times$ PBS, washed twice 5 min each with $1 \times$ PBS $+0.2 \%$ Triton X-100 (PBST), and fixed with 4\% PFA for $3 \mathrm{~h}$ at room temperature. Embryos were washed three times $\times 10$ min with PBST, dehydrated by incubation in increasing concentrations of methanol in PBS $\times$ $1\left(10 \%, 30 \%, 50 \%\right.$, and $80 \%, 1 \mathrm{~h}$ each), and stored overnight at $4^{\circ} \mathrm{C}$ in bleaching solution made of $6 \% \mathrm{H}_{2} \mathrm{O}_{2}$ and $80 \%$ methanol in $1 \times$ PBS. Embryos were then washed three times $\times 1 \mathrm{~h}$ with $80 \%$ methanol in $1 \times$ PBS, rehydrated with decreasing methanol concentrations in PBS $\times 1$ $(50 \%, 30 \%$, and $10 \%, 1 \mathrm{~h}$ each), washed three times $\times 30$ min with PBST, and blocked overnight at $4^{\circ} \mathrm{C}$ with $2 \%$ milk powder + PBST (PBSMT). Following $3 \mathrm{~d}$ incubation with primary mouse antiNeurofilament antibody (2H3, DSHB, 1:200 in PBSMT) at $4^{\circ} \mathrm{C}$, embryos were washed six times with PBSMT $\times 1 \mathrm{~h}$ and incubated overnight at $4^{\circ}$ $\mathrm{C}$ with secondary HRP-coupled goat anti-mouse antibody (1:200) in PBSMT $+2 \%$ goat serum. Embryos were washed four times with $\mathrm{PBSMT}+2 \%$ goat serum $\times 1 \mathrm{~h}$, then two times with PBST $\times 1 \mathrm{~h}$, and incubated for $30 \mathrm{~min}$ in $0.1 \mathrm{M}$ Tris, $\mathrm{pH}$ 7.5, + DAB (Sigma Millipore, 1 tablet per $15 \mathrm{ml}$ ) prefiltered using a $0.45 \mu \mathrm{m}$ filter. Development was performed by addition of $\mathrm{H}_{2} \mathrm{O}_{2}$ to the DAB solution $\left(0.03 \% \mathrm{H}_{2} \mathrm{O}_{2}\right.$ final concentration), and stopped with two $1 \times$ PBS washes $\times 2 \mathrm{~min}$, after fixation with $4 \%$ PFA for $15 \mathrm{~min}$, and additional three $1 \times$ PBS washes $\times$ 5 min.

For whole-mount TH staining, embryos at E13.5 were collected, and their sympathetic ganglia were exposed by dissection. Embryos were fixed with $4 \%$ PFA overnight at $4^{\circ} \mathrm{C}$, washed with $1 \times \mathrm{PBS}+1 \%$ Tween$20(\mathrm{PBST}) \times 10 \mathrm{~min}$, and incubated for $2 \mathrm{~h}$ in $0.9 \% \mathrm{NaCl}$ made in $1 \times$ PBS. Dehydration was conducted as described for the antiNeurofilament staining (but 15 min each instead of $1 \mathrm{~h}$ ), followed by bleaching in $6 \% \mathrm{H}_{2} \mathrm{O}_{2}+20 \%$ DMSO in $80 \%$ methanol in $1 \times$ PBS. Embryos were rehydrated (15min each), washed, blocked for $3 \mathrm{~h}$ at room temperature with $1 \times \mathrm{TBS}$, $\mathrm{pH} 7.4,+1 \%$ Tween-20 (TBST) $+5 \%$ milk powder (TBSMT), then incubated for $2 \mathrm{~d}$ at $4^{\circ} \mathrm{C}$ in primary rabbit anti-TH antibody (Abcam, 1:200, in TBSMT $+5 \%$ DMSO). After 6 washes $\times 1 \mathrm{~h}$ with TBST, embryos were incubated overnight at $4^{\circ} \mathrm{C}$ with secondary HRP-coupled goat anti-rabbit antibody (1:200) in TBST $+2 \%$ goat serum, then washed five times $\times 1 \mathrm{~h}$ with TBST, followed by $1 \mathrm{~h}$ wash with $1 \times$ TBS. Development was performed as described for the anti-Neurofilament staining.

\section{Golgi staining}

Adult (2-3 months old) mouse brains were processed for Golgi staining as previously described (FD Rapid GolgiStain Kit, catalog \#PK401, FD NeuroTechnologies) for $10 \mathrm{~d}$ (Tran et al., 2009). After incubation, all brains were blocked and embedded in OCT embedding medium 
(Tissue-Tek, catalog \#4583). Sagittal sections $(100 \mu \mathrm{m})$ were cut with a cryostat (Microm International, HM 505E) and mounted on charged microscopes slides (Diamond white glass). Staining procedures were followed exactly as described (FD NeuroTechnologies). Only layer 5 pyramidal neurons from the medial somatosensory cortex were included in our analyses. Differential interference contrast $z$-stack images were taken with an Axio Examiner Z1, Yokogawa spinning disk microscope (Carl Zeiss).

\section{Confocal images}

High-resolution confocal $z$-stack images of MAP2-immunostained neurons were taken using an Axio Examiner Z1, Yokogawa spinning disk microscope (Carl Zeiss) with an oil-immersion $63 \times$ (NA 1.4) objective (PlanApo, Carl Zeiss). High-resolution confocal $z$-stack images of GFPpositive cortical neurons from WT Thy1-EGFP, PlxnA4 ${ }^{\text {KRK-AAA }}$; Thy1EGFP and PlxnA4 $4^{+/ K R K-A A A} /$ Farp2 +/-; Thy1-EGFP animals were taken using an LSM510 META microscope (Carl Zeiss). Series of optical sections were acquired in the $z$ axis at $1 \mu \mathrm{m}$ intervals through individual layer 5 pyramidal neurons. Maximum projections of fixed images were analyzed using Fiji (is just ImageJ) software.

\section{Quantification of dendritic arborization}

Dendritic arborization was analyzed with the following parameters:

Sholl analysis. All Golgi-labeled $z$-stack images were reconstructed using Fiji and Adobe Photoshop CS6, and all analyses were performed using the Fiji Sholl Analysis plugin (Ferreira et al., 2014). For cortical basal dendrite Sholl analysis, the starting radius is $10 \mu \mathrm{m}$ and the ending radius is $60 \mu \mathrm{m}$ from the center of the neuron soma; the interval between consecutive radii is $5 \mu \mathrm{m}$ (Mlechkovich et al., 2014).

Total dendritic length. The total dendritic length was measured using the ImageJ plugin NeuronJ (http://www.imagescience.org/meijering/ software/neuronj/), calculated in microns $(\mu \mathrm{m})$.

Dendritic complexity index (DCI). Dendritic order is defined as follows: primary dendrites were traced from the cell soma to the tip of the entire dendritic length, and secondary and tertiary dendrites were traced from the tip to the dendritic branch point using Adobe Photoshop software. Dendrites that were $<10 \mu \mathrm{m}$ from the cell soma were disregarded. The DCI was calculated using the following formula: ( $\Sigma$ branch tip orders + \# branch tips)/(\# primary dendrites) $\times$ (total arbor length) (Lom and Cohen-Cory, 1999; Peng and Tran, 2017).

For each parameter analyzed, at least 30-40 neurons were measured per condition, from three or four independent cultures/mice.

\section{Western blot}

For Figure $1 B, C$, tissues were lysed with RIPA buffer (50 mu Tris, pH 7.4, $159 \mathrm{~mm} \mathrm{NaCl}, 1 \% \mathrm{NP} 40,0.1 \%$ SDS, 0.5\% deoxycholate, 1 mm EDTA in $\mathrm{ddH}_{2} \mathrm{O}$ ) supplemented with 1:25 cOmplete Protease Inhibitor Cocktail (Roche Diagnostic, catalog \#11697498001) and 1:200 PMSF. Samples then underwent two cycles of 10-min-long incubation on ice followed by $10 \mathrm{~s}$ of vortexing. After $14,000 \times g$ centrifugation for $15 \mathrm{~min}$ at $4^{\circ} \mathrm{C}$, supernatant was collected and protein concentration was measured using Pierce BCA Protein Assay Kit (Thermo Fisher Scientific, catalog \#23227) and Gen5 3.04 software (BioTek). Sample buffer X5 (125 mM Tris $\mathrm{HCl}, \mathrm{pH} 6.8,25 \% \beta$-mercaptoethanol, $43.5 \%$ glycerol, $10 \%$ SDS, $0.05 \%$ bromophenol blue) was added to the samples that were further denatured by boiling at $95^{\circ} \mathrm{C}$ for $5 \mathrm{~min}$. Samples were separated by SDS-PAGE and electrophoretically transferred onto nitrocellulose membranes. Nonspecific antigen sites were blocked using 5\% milk in TBS (10

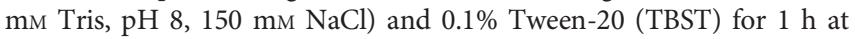
room temperature. Antigen detection was performed by overnight incubation with appropriate antibodies: 1:1000 rabbit monoclonal Plexin-A4 (catalog \#C5D1) anti-mouse (Cell Signaling Technology, catalog \#3816), 1:1000 rabbit monoclonal Nrp1 (catalog \#D62C6) anti-mouse (Cell Signaling Technology, catalog \#3725), and 1:30,000 mouse anti-actin antibody C4 (MP Biomedicals, catalog \#08691001). Antibodies were diluted in $5 \%$ BSA in TBST $+0.05 \%$ sodium azide. Membranes were then washed and incubated for $1 \mathrm{~h}$ in at room temperature in goat antirabbit HRP (Jackson ImmunoResearch Laboratories, 111-035-003) or goat anti-mouse (Jackson ImmunoResearch Laboratories, 115-035-003) secondary antibodies, following incubation in WesternBright HRP- substrate enhanced chemiluminescence (Advansta, catalog \#K-12 045D20) and exposure to Super RX-N X-Ray films (Fujifilm, catalog \#47 410). Films were scanned and band intensities were quantified using ImageJ (Fiji) software.

For Figure $1 D$, mice were sacrificed using a $\mathrm{CO}_{2}$ chamber. The cortices were immediately removed from the skull and homogenized in RIPA buffer containing protease inhibitors (Roche cOmplete Ref. 11697498001). Homogenates were cleared by centrifugation at $500 \times g$ twice for $7 \mathrm{~min}$, and protein concentration was determined using the Bradford protocol (Bradford, 1976); samples were then boiled in gel-loading buffer and separated using SDS-PAGE, 10\% for PlxnA4 (catalog \#C5D1, Cell Signaling Technology), and IgG. Proteins were transferred to nitrocellulose membranes (Bio-Rad, catalog \#1620097) and blocked with 5\% nonfat milk in TBS with $0.05 \%$ Tween, for $1 \mathrm{~h}$ at room temperature. The membranes were incubated with primary antibodies (overnight at $4^{\circ} \mathrm{C}$ ), washed, and reincubated with the secondary HRP-conjugated antibody (1:2000 dilution for anti-rabbit, GE Healthcare, NA934, lot \#6969611; 1:2000 anti-mouse GENXA931; $1 \mathrm{~h}$ at room temperature). Peroxidase activity was visualized by using enhanced chemiluminescence kit (catalog \#32106, Thermo Fisher Scientific). Enhanced chemiluminescence signal was exposed to BioBlue Lite films XR8813. Membranes were reprobed with a monoclonal antibody against IgG for control of protein loading. The images were quantified using Fiji software.

\section{Coimmunoprecipitation assay}

Mice were sacrificed using a $\mathrm{CO}_{2}$ chamber, and the cortices were immediately removed from the skull and homogenized in RIPA-modified buffer (TBS $1 \times, 10 \%$ glycerol, $1 \%$ Triton X-100, and 1\% NP40) supplemented with protease inhibitors. Briefly, the homogenates were centrifuged at $14,000 \times g$ for $10 \mathrm{~min}$, and $500 \mu \mathrm{g}$ of total protein from the supernatants was precleared with Protein G Sepharose 4 Fast Flow (GE Healthcare, cata$\log \# \mathrm{GE} 17-0618-01)$ for $2 \mathrm{~h}$ at $4^{\circ} \mathrm{C}$. After centrifugation, the precleared supernatants were incubated with anti-Nrp1 (R\&D Systems, catalog \#AF566) at $4^{\circ} \mathrm{C}$ for overnight. Then $10 \mu$ l protein Protein G Sepharose 4 Fast Flow was added and incubated at $4^{\circ} \mathrm{C}$ for $4 \mathrm{~h}$. The immunoprecipitates were washed with ice-cold RIPA-modified buffer, eluted with SDS sample buffer, and analyzed by Western blot. Membranes where then probed with anti-PlxnA4 (catalog \#C5D1, Cell Signaling Technology) antibody. As a protein loading control, we first incubated the membranes with the secondary antibody anti-goat alone (primary antibody from goat).

\section{Cell-surface biotinylation assay}

Cortical neurons from E13.5 embryos were extracted and grown in culture. At 7 DIV, a cell surface biotinylation assay was performed using a commercially available kit (Pierce Cell Surface Protein Isolation Kit, Thermo Fisher Scientific, catalog \#89881). Eluates were subjected to immunoblot analysis using a Plexin-A4 antibody (Cell Signaling Technology, catalog \#3816), as described above in Western blot. Cell lysates obtained following the biotinylation process were used as loading controls, and the total mass of proteins between $\sim 120$ and $260 \mathrm{kDa}$ was used for quantification and normalization purposes.

\section{Real-time RT-PCR}

Heads of E13.5 embryos were collected and subjected to RNA extraction. Briefly, heads were homogenized in TRI reagent (Sigma Millipore) and chloroform was added. Samples were mixed thoroughly, incubated at room temperature for $10 \mathrm{~min}$, centrifuged at $13,000 \mathrm{rpm}$ for $15 \mathrm{~min}$ at $4^{\circ}$ $\mathrm{C}$, and upper RNA-containing phase was collected. Precipitation of RNA was performed using isopropanol, after which samples were incubated at room temperature for $10 \mathrm{~min}$, and centrifuged at 13,000 rpm for $15 \mathrm{~min}$ at $4^{\circ} \mathrm{C}$. Cold $75 \%$ ethanol was added to the RNA pellet, and samples were stored overnight at $-80^{\circ} \mathrm{C}$. The next day, ethanol was decanted, and samples were incubated at $65^{\circ} \mathrm{C}$ for $5 \mathrm{~min}$ to remove all traces of ethanol. RNA pellet was liquidized with $30 \mu \mathrm{l}$ ultrapure DNase/RNase free $\mathrm{H}_{2} \mathrm{O}$, and RNA concentration was measured using NanoDrop spectrophotometer. cDNA was then synthesized from $1 \mu \mathrm{l} / \mathrm{RNA}$ sample using an iScript kit (Bio-Rad, catalog \#1708891), and PlxnA4 expression levels were measured by qRT-PCR reaction using KAPA SYBR FAST ABI prism qPCR kit (Kapa Biosystems, catalog \#KK4605). Primers (5' $\left.3^{\prime}\right)$ were as follows: PlxnA4, GCAAGAACTTGATCCCGCCT and 
GTCACGGTGCATGGTTTCTC; Actb, GGCTGTATTCCCCTCCA TCG and CCAGTTGGTAACAATGCCATGT. Results were subjected to relative quantification analysis $\left(\Delta \Delta \mathrm{C}_{\mathrm{t}}\right)$ using Microsoft Excel software.

\section{Experimental design and statistical analyses}

For sequencing analysis of exon 20 in the PlxnA4 locus experiments, a total of 3 animals were used. For PlxnA4 mRNA qRT-PCR analysis, a total of 6 animals/genotype were used, and a Student's $t$ test was performed. For Western blot analysis of Plexin-A4, Nrp1, and actin proteins in E13.5 DRGs and cortices extracted from PlxnA4 ${ }^{\text {KRK-AAA }}$, WT littermate, and PlxnA4 KO, a total of 4 mice/genotype were used from three independent experiments. For Western blot analysis of Nrp1:Plexin-A4 interaction, cerebral cortices were extracted from PlxnA4 $4^{K R K-A A A}$, WT littermate, and PlxnA4 KO, a total of 3 animals/genotype were used from three independent experiments. For analysis of the expression pattern of the PlxnA4 protein, primary cortical neurons obtained from WT, $P l x n A 4^{K R K-A A A}$, and PlxnA4 KO embryos were immunostained using a Plexin-A4 antibody and a total of three independent cultures/genotypes. For analysis of Plexin-A4's cell-surface expression level, three independent cell-surface biotinylation experiments were conducted and followed by an immunoblot analysis using a Plexin-A4 antibody. For the analysis of Sema3A-mediated growth cone collapse using DRG explants obtained from WT, PlxnA4 ${ }^{\text {KRK-AAA }}$, and Farp 2 KO embryos, a total of at least 300 growth cones/group from three independent experiments were analyzed, and two-way ANOVA with post hoc Tukey test was performed. For the Sema3A-collagen axonal repulsion assay using DRG explants obtained from WT, PlxnA4 ${ }^{K R K-A A A}$, and Farp2 KO embryos, a total of at least 8 DRGs/genotype from three independent experiments were used, and a Student's $t$ test was performed. For Sema3A-treatment in primary cortical neuron culture obtained from WT, PlxnA4 KO, PlxnA4 ${ }^{\text {KRK-AAA }}$, and Farp2 KO, a total of three independent cultures/genotype were used, and a Student's $t$ test and a two-way ANOVA followed by post hoc Tukey test were performed. For Golgi-stained layer $\mathrm{V}$ cortical neurons obtained from WT, PlxnA4 KO, PlxnA4 $4^{K R K-A A A}$, Farp2 KO, and PlxnA4 $4^{+/ K R K-A A A}$ / $\mathrm{Farp2}^{+/-}$, a total of 3 animals/genotype were used. For analysis of layer $\mathrm{V}$ cortical neurons in WT, PlxnA4 $4^{K R K-A A A}$, and in PlxnA4 $4^{+/ K R K-A A A}$ / Farp $2^{+/-}$, using ThylGFP mice, a total of 3 animals/genotype were used. For quantification of dendritic morphologies using Sholl analysis, dendritic length, DCI, and number of dendritic tips, one-way ANOVA and post hoc Tukey test were performed. For the analysis of the pattern of cutaneous sensory axons projections in WT, PlxnA4 ${ }^{\text {KRK-AAA }}$, Farp2 KO, or PlxnA4 KO E12.5 embryos, using a Neurofilament antibody, a total of at least eight embryos/genotype from at least three independent experiments were used. For the analysis of sympathetic axon projections in WT, PlxnA4 ${ }^{\text {KRK-AAA }}$, Farp2 KO, or PlxnA4 KO E13.5 embryos, a total of at least 10 embryos/genotype from at least three independent experiments were used. For the analysis of the anterior commissure in WT, PlxnA4 ${ }^{K R K-A A A}$, Farp2 KO, or PlxnA4 KO, using hematoxylin staining, a total of at least seven brains/genotype from at least three independent experiments were used. For the analysis of the Rac signaling in Sema3Adependent growth cone collapse of phalloidin-rhodamine-stained DRG neurons obtained from WT and PlxnA4 ${ }^{\text {KRK-AAA }}$ DRGs, a total of at least 300 growth cones/group from three independent experiments were analyzed, and a two-way ANOVA with post hoc Tukey test was performed. For the analysis of the Rac signaling inhibition (using EHT) in Sema3Adependent dendritic growth in WT cortical primary neurons, a total of three independent cultures were used, and a two-way ANOVA followed by post hoc Tukey test was performed. For the analysis of Sema3A-dependent dendritic arborization in the Farp2 KO with Rac inhibitor, three independent cultures were performed, and a two-way ANOVA followed by post hoc Tukey test was performed. For the Western blot of Rac1 knockdown experiments, using a specific Racl siRNA, three independent primary WT neuron cultures were used, and a Student's $t$ test was performed. For Sema3A treatment in the Rac1 knockdown experiments, a total of three independent primary WT cortical neuron cultures were used, and two-way ANOVA followed by post hoc Tukey test was performed. For the pulldown assay showing the effect of 30 min stimulation of Sema3A on Rac1 activation, three independent WT, PlxnA4 ${ }^{\text {KRK-AAA }}$, and Farp 2 KO primary neuron cultures were used, and Student's $t$ test and one-way ANOVA were performed. For DRG growth cone collapse and cortical neuron dendritic arborization quantifications, data obtained were analyzed using GraphPad Prism Software. For DRG axonal repulsion, data were analyzed using Microsoft Excel software. Data are shown as mean \pm SEM.

\section{Results \\ Generation of the PlxnA4 ${ }^{\text {KRK-AAA }}$ mutant and Farp2 KO mouse}

To explore the role of the KRK motif in the PlxnA4 cytoplasmic domain, we have generated by CRISPR the PlxnA4 $4^{K R K-A A A}$ line that harbors a KRK substitution to AAA in the endogenous PlxnA4 receptor. The modified genomic area $(220 \mathrm{bp})$ from tail genomic DNA was sequenced, and only the desired modification was detected. The mutation had no effect on PlxnA4 mRNA or total protein levels, as shown using biochemical analysis (Fig. $1 A$ and Fig. $1 B, C$, respectively). Moreover, we have not detected any change in the levels of the PlxnA4 coreceptor (Fig. $1 B, C$ ), nor change in the association of Nrp1 with PlxnA4 in the PlxnA4 $4^{\text {KRK-AAA }}$ mutants (Fig. 1D). Furthermore, the KRK to AAA substitution did not affect the cell surface expression of PlxnA4 on cortical neurons, as revealed by detection of the biotinylated receptor (Fig. $1 E, F)$, or its dendritic localization (Fig. $1 G$ ). Overall, our analysis shows that the KRK to AAA mutation did not alter the expression and cellular distribution of Plexin-A4. In parallel, we have generated a Farp2 KO mouse by CRISPR (Fig. $1 H$ ). Using PCR analysis of tail genomic DNA and reverse transcription analysis of progenies from the Farp2 $\mathrm{KO}$ line, we also demonstrated successful deletion of the Farp2 locus and its transcripts (Fig. 1I,J). For both mutant lines, the injected animals (F0) were crossed with WT CD1 mice three times. In all the experiments described below, WT littermate controls were used.

\section{DRG sensory axons from PlxnA4 ${ }^{K R K-A A A}$ and Farp2 KO animals are sensitive to Sema3 $A$ inhibition and repulsion} The PlxnA4 ${ }^{K R K-A A A}$ and the Farp2 mutant animals allowed us to systemically examine their role in Sema3A signaling using multiple in vitro assays. We first assessed the acute growth cone collapse assay. DRG axonal growth cones from E13.5 PlxnA4 ${ }^{K R K-A A A}$ homozygous mutant, grown for 2 DIV and visualized with phalloidin-rhodamine, were able to respond to Sema3A-mediated collapse in a dosage-dependent manner, similar to the WT control (Fig. $2 A, B$ ). In line with our results from the PlxnA4 ${ }^{K R K-A A A} \mathrm{mu}-$ tant neurons, DRG axonal growth cones from mice deficient of FARP2 GEF (Farp2 KO), which is a downstream target of the KRK motif in PlxnA4, collapsed at percentages comparable with the WT control following Sema3A treatment (Fig. 2A,B). Next, we tested the response to the prolonged Sema3A-mediated axonal repulsion in PlxnA4 $4^{K R K-A A A}$ and Farp2 mutant animals. For this, we cocultured DRG explants with COS1 cell aggregates either transfected with GFP (control) or a construct expressing Sema3A. Both DRG sensory axons from PlxnA4 $4^{\text {KRK-AAA }}$ and Farp2 KO explants were repelled from the Sema3A source, similar to WT DRG axons (Fig. 2D). Quantification of axonal repulsion using the proximal/distal axonal outgrowth ratio measurement as previously described (Yaron et al., 2005) showed no significant difference between PlxnA4 $4^{K R K-A A A}$ or Farp2 KO explants compared with their respective littermate WT controls in response to Sema3A repulsion (Fig. $2 E ; p=0.48$ ). These results suggest that the KRK motif in the PlxnA4 receptor and its downstream target FARP2 are dispensable for both Sema3A-mediated acute growth cone collapse and prolonged axonal repulsion. 


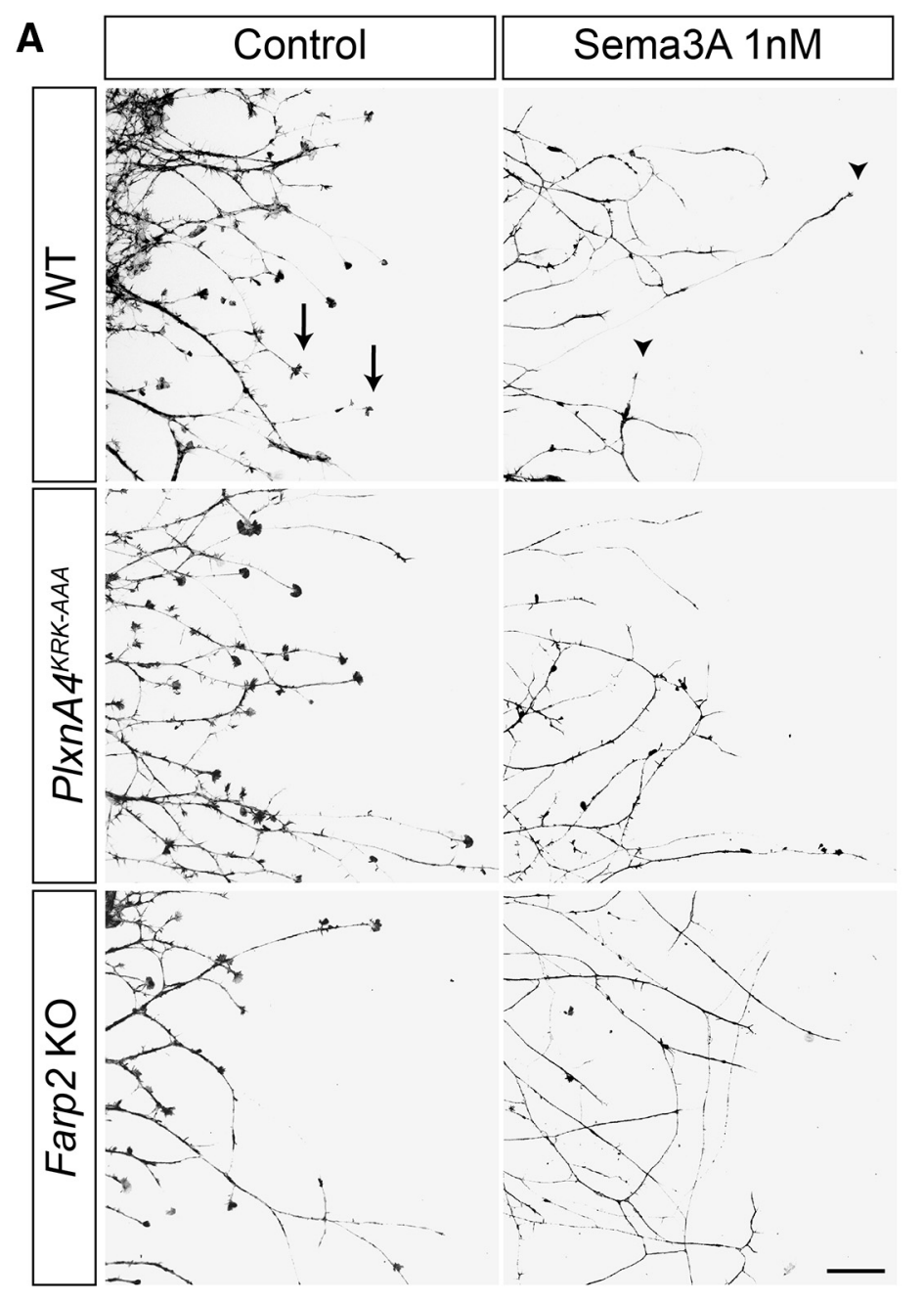

\section{B}

C
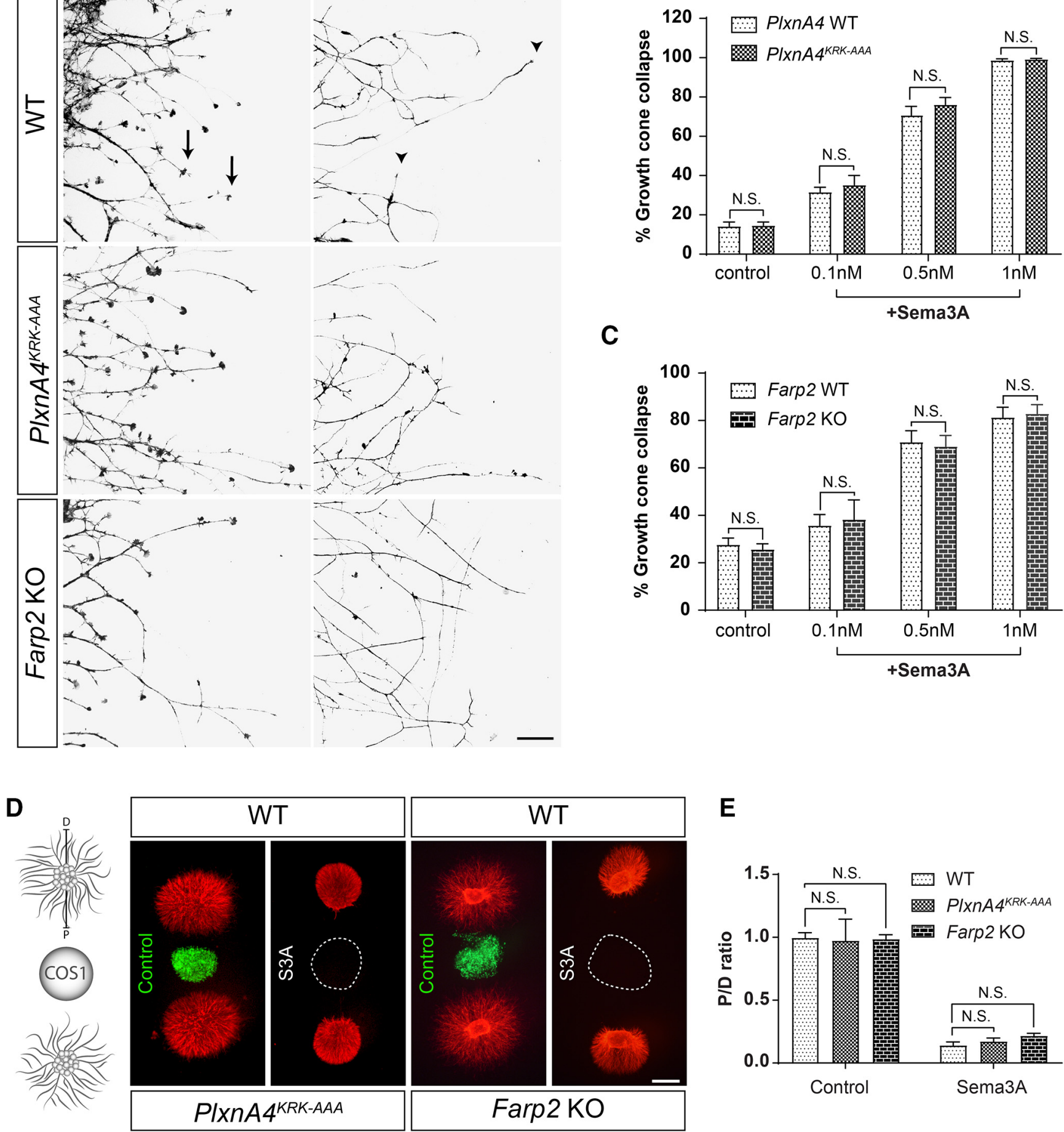

E

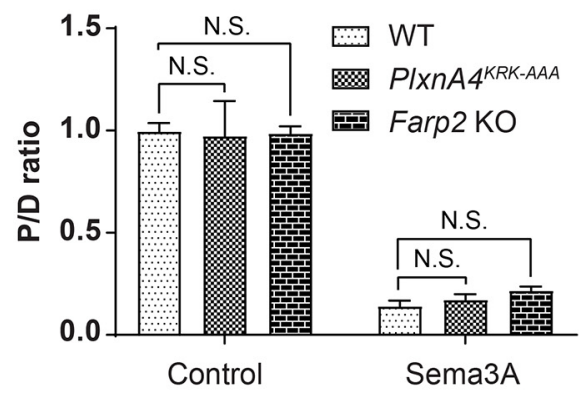

Figure 2. PlxnA KRK ${ }^{K A A A}$ and Farp2 KO DRG axons show intact Sema3A-dependent responses in vitro. A, DRG explants from WT, P/xnA4 KRK-AAA, and Farp2 KO E13.5 embry0s

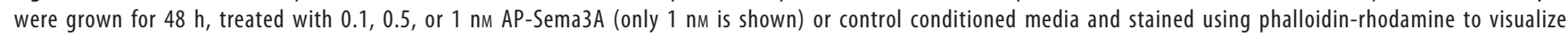
growth cone collapse. Arrows indicate intact growth cones. Arrowheads indicate collapsed growth cones. $\boldsymbol{B}, \boldsymbol{C}$, Quantification of collapse response as a mean percentage of collapsed growth cones out of the total \pm SEM. Statistical analysis: two-way ANOVA with post hoc Tukey test. Scale bar, $50 \mu \mathrm{m}$. D, DRG explants from E13.5 P/xnA4 KRK-AAA and WT littermates or Farp2 KO and WT littermates were cocultured in collagen droplets for $48 \mathrm{~h}$ with a COS1 aggregate either secreting myc-Sema3A (dashed circle) or expressing control PAY1-GFP (green). Cultures were visualized using anti-tubulin Class III immunostaining. $\boldsymbol{E}$, Quantification of axonal repulsion using the proximal/distal $(P / D)$ ratio, as indicated in the schematic representation of the collagen axonal repulsion assay in $\boldsymbol{D}$. Data are mean \pm SEM. Statistical analysis: Student's $t$ test; N.S., Non Significant. Scale bar, $500 \mu \mathrm{m}$. 


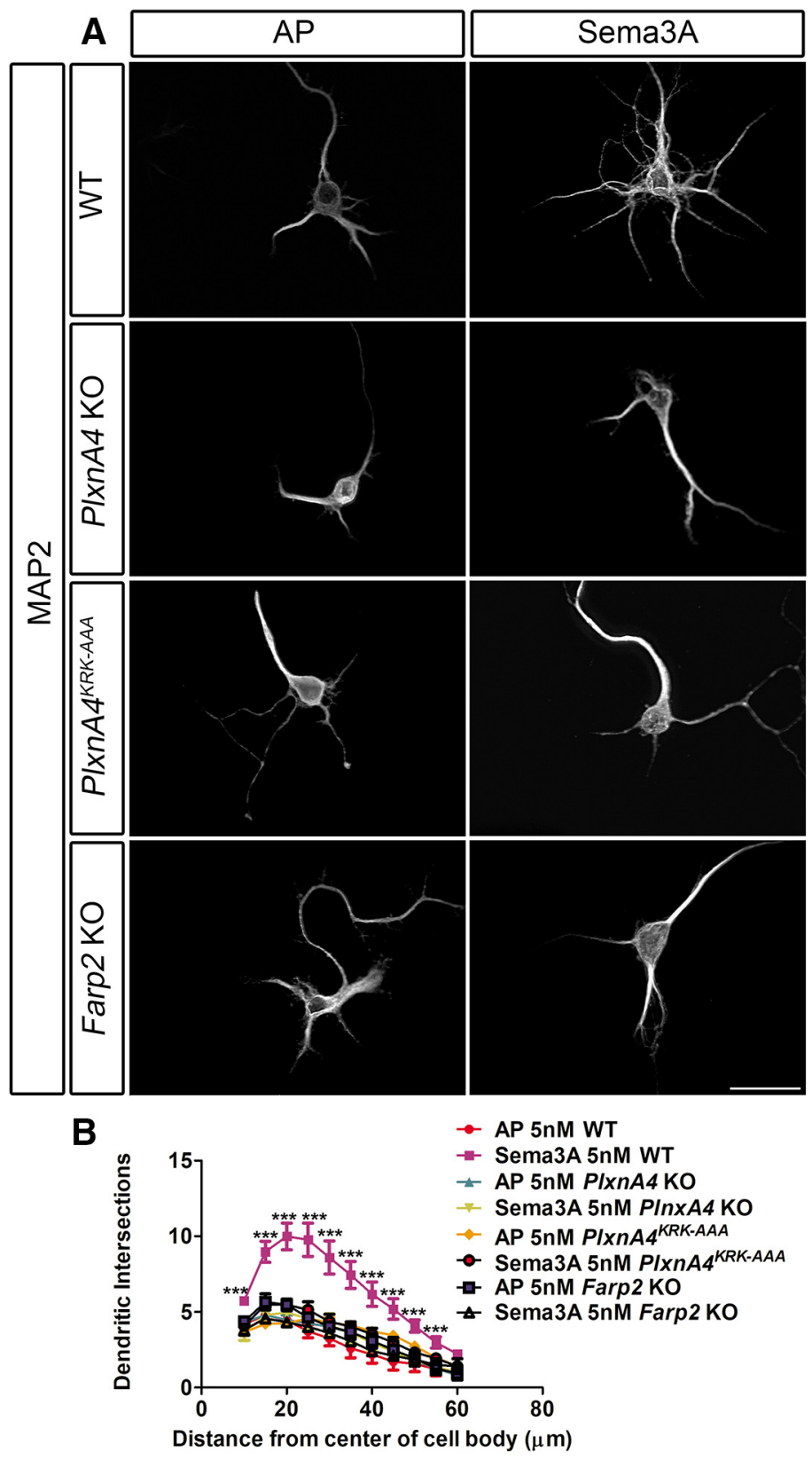

C
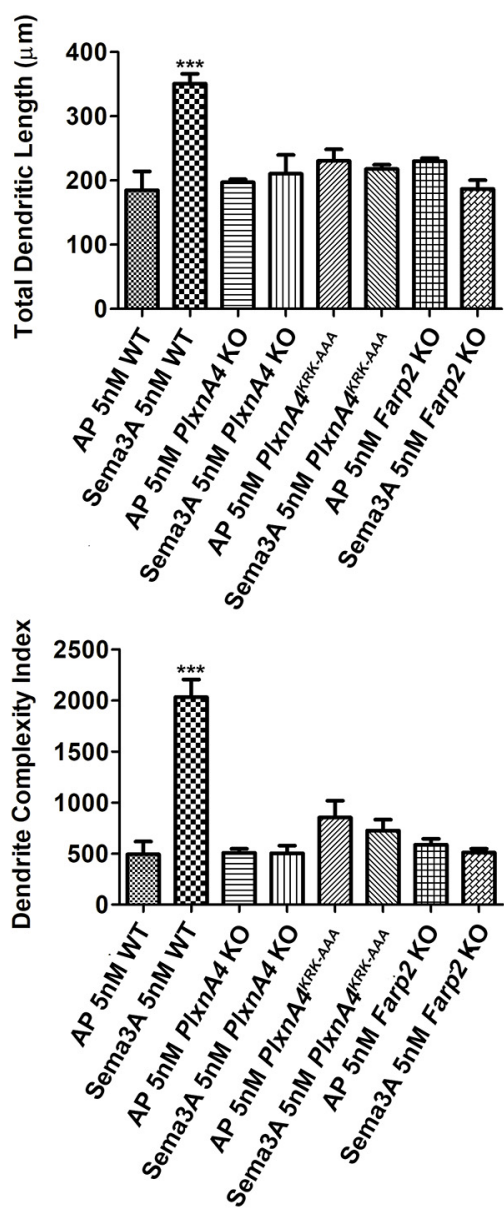

E

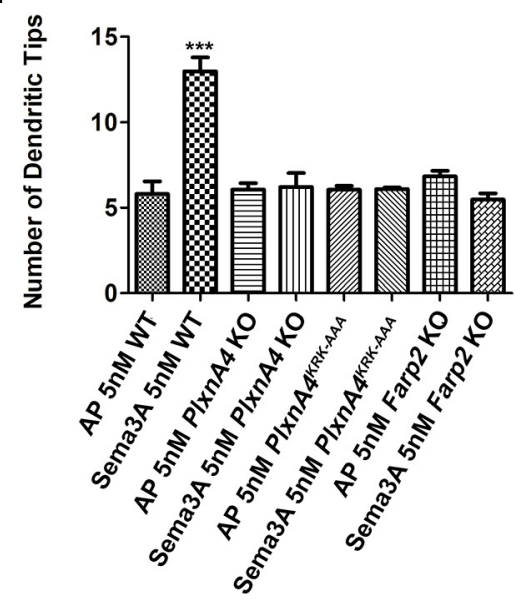

Figure 3. Both PlxnA $4^{K R K-A A A}$ and Farp2 KO neurons are nonresponsive to Sema3A-induced cortical neuron dendrite outgrowth in vitro. $\boldsymbol{A}$, Representative confocal micrographs of dissociated primary cortical neurons obtained from WT, PlxnA4 KO, PlxnA4 ${ }^{K R K-A A A}$, and Farp2 KO E13.5 embryos. The neurons were treated with AP or Sema3A 5 nM for 24 h. B-E, Sholl analysis quantified dendritic growth and branching in WT, PlxnA4 KO, PIxnA $4^{K R K-A A A}$, and Farp2 KO cortical neurons $(\boldsymbol{B})$, measuring the number of dendritic intersections, the total dendritic length $(\boldsymbol{C})$, the DCI (D), and the number of dendritic tips $(\boldsymbol{E})$. Data are mean \pm SEM from three independent cultures of each genotype. ${ }^{* * *} p<0.001$ (two-way ANOVA with post hoc Tukey test). Scale bar, $25 \mu \mathrm{m}$.

Cortical neurons from PlxnA4 ${ }^{K R K-A A A}$ and Farp2 KO animals are nonresponsive to Sema3A-induced dendrite elaboration

As Sema3A KO brains have previously been shown to display similar cortical neuron basal dendrite morphologic defects (Nakamura et al., 2017) similar to those observed in PlxnA4 KO (Tran et al., 2009; Mlechkovich et al., 2014; Peng and Tran, 2017), we asked whether the KRK motif of PlxnA4 and its downstream target FARP2 are required for Sema3A-mediated cortical dendrite branching. Embryonic cortical neurons at E13.5 were dissociated from WT, PlxnA4 $4^{K R K-A A A}$ mutant, PlxnA4 KO, and Farp2 KO animals as previously described (Tran et al., 2009; Mlechkovich et al., 2014; Peng and Tran, 2017) and grown for 5 DIV followed by Sema3A treatment for $24 \mathrm{~h}$ and fixation for anti-MAP2 immunocytochemistry to visualize all dendrites (Fig. 3A). Using four different measurements, 

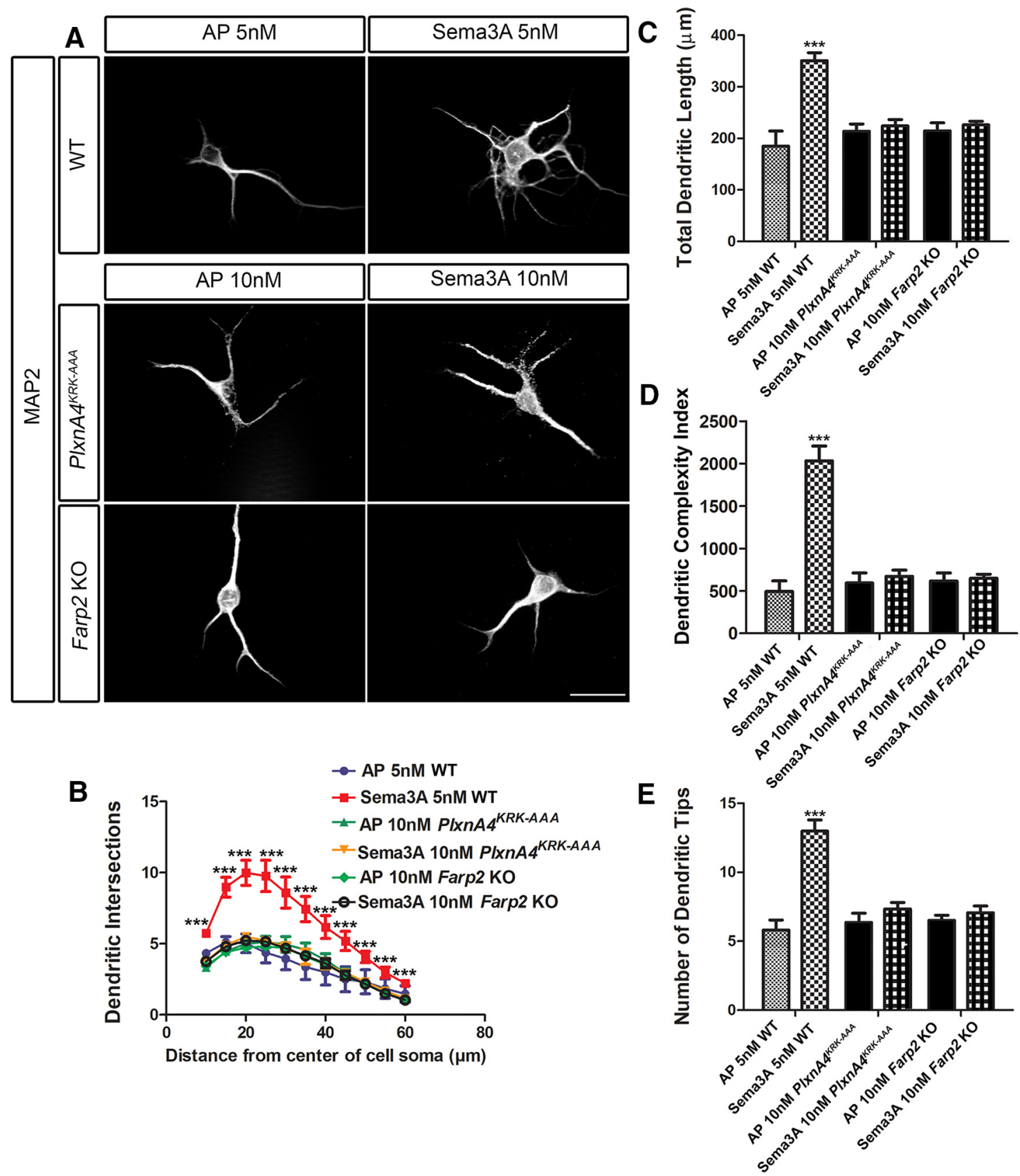

Figure 4. Both PlxnA4 ${ }^{K R K-A A A}$ and Farp2 KO cortical neurons are nonresponsive, even to higher concentrations of Sema3A. A, Representative confocal micrographs of dissociated primary cortical neurons obtained from WT, PlXnA4 $4^{K R K-A A A}$, and Farp2 KO E13.5 embryos. The WT neurons were treated with AP or Sema3A 5 nM, and the PlxnA4 $4^{K R K-A A A}$ and Farp2 KO neurons were treated with AP or Sema3A $10 \mathrm{~nm}$ for 24 h. $\boldsymbol{B}-\boldsymbol{E}$, Sholl analysis quantified dendritic growth and branching in WT, PlxnA4RK ${ }^{K R A A}$, and Farp2 KO cortical neurons $(\boldsymbol{B})$, measuring the number of dendritic intersections, the total dendritic length $(\boldsymbol{C})$, the $\mathrm{DCl}(\boldsymbol{D})$, and the number of dendritic tips $(\boldsymbol{E})$. Data are mean \pm SEM from three independent cultures of each genotype. ${ }^{* * *} p<0.001$ (twoway ANOVA with post hoc Tukey test). Scale bar, $25 \mu \mathrm{m}$.

we quantified the number of dendritic intersections (by Sholl analysis; Fig. $3 B$ ), the total dendritic length (Fig. $3 C$ ), the DCI (Fig. 3D), and the average number of dendritic tips (Fig. $3 E$ ). Consistent with previous results, Sema3A-treated WT neurons showed increased number of dendrites compared with the control-treated (Student's $t$ test, Sholl: $10 \mu \mathrm{m}: t=4.775, p=0.0088$; $15 \mu \mathrm{m}: t=5.422, p=0.0056 ; 20 \mu \mathrm{m}: t=5.706, p=0.0047 ; 25 \mu \mathrm{m}$ : $t=5.119, p=0.0069, \quad 30 \mu \mathrm{m}: t=4.443, p=0.0113 ; 35 \mu \mathrm{m}: t=$ 4.458, $p=0.0112 ; 40 \mu \mathrm{m}: t=3.999, p=0.0161 ; 45 \mu \mathrm{m}: t=3.934$, $p=0.0170 ; 50 \mu \mathrm{m}: t=3.807, p=0.0190 ; 55 \mu \mathrm{m}: t=2.952, p=$ $0.0419 ; 60 \mu \mathrm{m}: t=2.910, p=0.0437$; length: $t=5.022, p=0.0074$; DCI: $t=7.175, p=0.0020$; and dendritic tips: $t=6.553, p=$
0.0028). Next, we asked whether neurons obtained from PlxnA4 ${ }^{\text {KRK-AAA }}$, Farp2 KO, and PlxnA4 KO show sensitivity to Sema3A treatment. We found that PlxnA4 ${ }^{K R K-A A A}$ mutant and Farp2 $\mathrm{KO}$ neurons were insensitive to Sema3A stimulation, similarly to the PlxnA4 KO (two-way ANOVA: Sholl: $10 \mu \mathrm{m}$ : $p=0.0023, F=7.554 ; 15 \mu \mathrm{m}: p<0.0001, F=14.41 ; 20 \mu \mathrm{m}: p<$ $0.0001, F=18.04 ; 25 \mu \mathrm{m}: \quad p<0.0001, \quad F=15.59 ; 30 \mu \mathrm{m}: p=$ $0.0002, F=12.09 ; 35 \mu \mathrm{m}: p=0.0001, F=13.05 ; 40 \mu \mathrm{m}: p=0.0004$, $F=10.59 ; 45 \mu \mathrm{m}: p=0.0004, F=10.72 ; 50 \mu \mathrm{m}: p=0.0015, F=$ 8.284; $55 \mu \mathrm{m}: p=0.0422, F=3.437$; length: $p=0.0001, F=13,64$; DCI: $p<0.0001, F=27$; dendritic tips: $p<0.0001, F=25.50$ ). Similarly, there were no significant differences in dendritic 

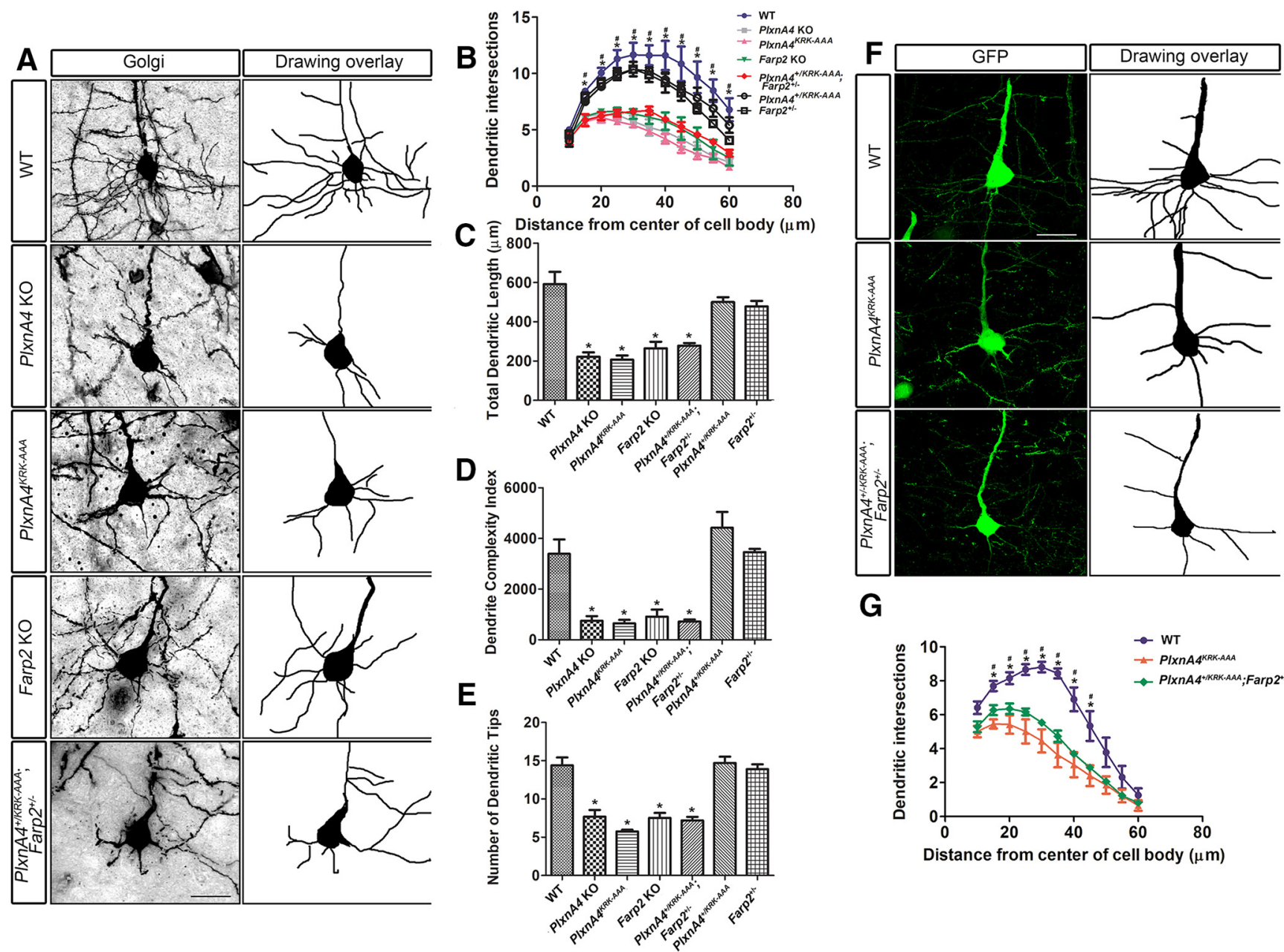

G

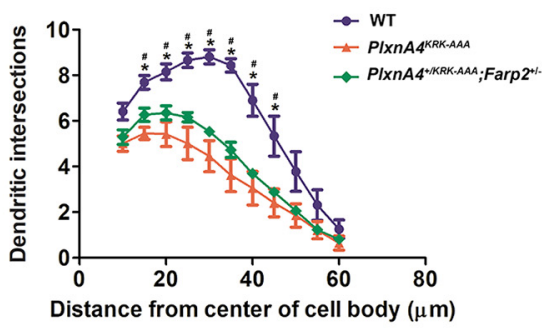

Figure 5. Reduced basal dendritic arborization in layer 5 cortical neurons from PlxnA4RK-AAA and Farp2 KO adult animals. $A$, Golgi-stained images of brain (2- to 3-month-old) sections of WT, PIXnA4 K0, PlXnA4 ${ }^{K R K-A A A}$, Farp2 KO, and PlXnA4 ${ }^{+/ K R K-A A A} / \mathrm{Farp2}^{+/-}$. B. Sholl analysis quantification revealed reduced and altered branching patterns of basal dendrites from layer V cortical

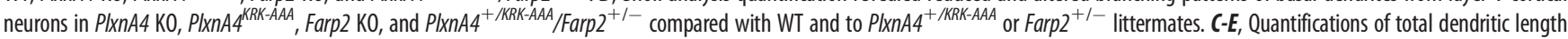
$(\boldsymbol{C}), \mathrm{DCl}(\boldsymbol{D})$, and dendritic tips $(\boldsymbol{E})$ in all genotype analyzed. Data are mean \pm SEM. ${ }^{*} p<0.05$ (one-way ANOVA followed by post hoc Tukey test). $\boldsymbol{F}, \boldsymbol{G}$, Representative images of the layer 5 cortical neurons and Sholl analysis obtained from a WT, P/xnA4 ${ }^{\text {KRK-AAA }}$, and PIXnA4 ${ }^{+/ K R K-A A A} /$ Farp2 ${ }^{+/-}$Thy1-GFP mice. Statistical analysis: one-way ANOVA followed by post hoc Tukey test. Data are mean \pm SEM; $n=3$ brains/genotype. ${ }^{*} p<0.05$, statistical difference between WT and PlxnA $4^{\text {KRK-AAA }}$ mutant or PlxnA4 K0 or Farp2 KO in $\boldsymbol{B}$ and $\boldsymbol{G}$. ${ }^{\#} p<0.05$, statistical difference

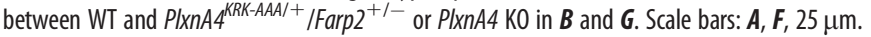

morphology as measured by Sholl analysis, dendritic length, DCI, and average number of dendritic tips between AP-treated WT neurons versus AP or Sema3A-treated PlxnA4 ${ }^{K R K-A A A}$ mutant, PlxnA4 KO, and Farp2 KO neurons. In addition, we tested whether higher Sema3A concentration could induce dendrite growth in PlxnA4 $4^{K R K-A A A}$ mutant and Farp2 KO neurons. $P l x n A 4^{K R K-A A A}$ and Farp2 KO cortical neurons were treated with $10 \mathrm{~nm}$ of Sema3A for $24 \mathrm{~h}$. We analyzed the dendritic morphology measured by Sholl analysis, dendritic length, DCI, and dendritic tips. We found that both PlxnA4 ${ }^{K K-A A A}$ and Farp2 $\mathrm{KO}$ cortical neurons remained nonresponsive, even to the higher concentration of Sema3A (two-way ANOVA, Sholl: $10 \mu \mathrm{m}: p=0.0075, F=7.565 ; 15 \mu \mathrm{m}: p=0.0002, F=19.6 ; 20 \mu \mathrm{m}$ : $p<0.0001, \quad F=22.55 ; 25 \mu \mathrm{m}: \quad p=0.0001, \quad F=20.66 ; \quad 30 \mu \mathrm{m}:$ $p=0.0005, F=15.11 ; 35 \mu \mathrm{m}: p=0.0006, F=14.54 ; 40 \mu \mathrm{m}: p=$ $0.0018, F=11.25 ; 45 \mu \mathrm{m}: p=0.0016, F=11.49 ; 50 \mu \mathrm{m}: p=0.0025$, $F=10.30 ; 55 \mu \mathrm{m}: p=0.0085, F=7.27 ; 60 \mu \mathrm{m}: p=0.0132, F=$ 6.342; length: $p=0.0007, F=13.92$; DCI: $p<0.0001, F=28.55$; dendritic tips: $p=0.0002, F=18.58$ ) (Fig. 4). Therefore, in contrast to DRG sensory neurons, these results suggest that PlxnA4 ${ }^{\text {KRK-AAA }}$ mutant and Farp2 KO cortical neurons are selectively nonresponsive to Sema3A-mediated dendrite elaboration.
PlxnA4 ${ }^{\text {KRK-AAA }}$ and Farp2 KO animals exhibit reduced basal dendritic arborization in layer 5 cortical neurons, but not axonal repulsion defects in vivo

Next, we asked whether PlxnA4 $4^{\text {KRK-AAA }}$ homozygous mutant and Farp $2 \mathrm{KO}$ embryos display dendritic elaboration phenotypes in layer 5 pyramidal neurons in vivo. We performed Golgi staining to uniformly label all dendritic processes in adult brains of WT, PlxnA4 ${ }^{K R K-A A A}$ mutant, PlxnA4 KO, and Farp2 KO animals. We observed severe reduction in the basal dendritic arbors of layer 5 cortical neurons in the PlxnA4 ${ }^{\text {KRK-AAA }}$ mutant, PlxnA4 $\mathrm{KO}$, and Farp2 KO brains compared with the WT (Fig. 5A). As revealed by Sholl analysis, there was a significant decrease in dendritic intersections between WT and PlxnA4 ${ }^{K R K-A A A}$ mutant or PlxnA4 KO or Farp2 KO (Fig. 5B; Sholl: $15 \mu \mathrm{m}: p<0.0001$, $F=50.83 ; 20 \mu \mathrm{m}: p<0.0001, F=32.55 ; 25 \mu \mathrm{m}: p<0.0001$, $F=23.39 ; 30 \mu \mathrm{m}: p=0.0001, F=18.50 ; 35 \mu \mathrm{m}: p<0.0001, F=$ 20.56; $40 \mu \mathrm{m}: p=0.0006, F=13.08 ; 45 \mu \mathrm{m}: p=0.0017, F=10.16$; $50 \mu \mathrm{m}: p=0.0014, F=10.67 ; 55 \mu \mathrm{m}: p<0.0003, F=15.59 ; 60 \mu \mathrm{m}$ : $p=0.0009, F=11.79)$. In addition, the total dendritic length, the DCI, and average number of dendritic tips for PlxnA4 KRK-AAA mutant, PlxnA4 KO, and Farp2 KO were lower compared with 

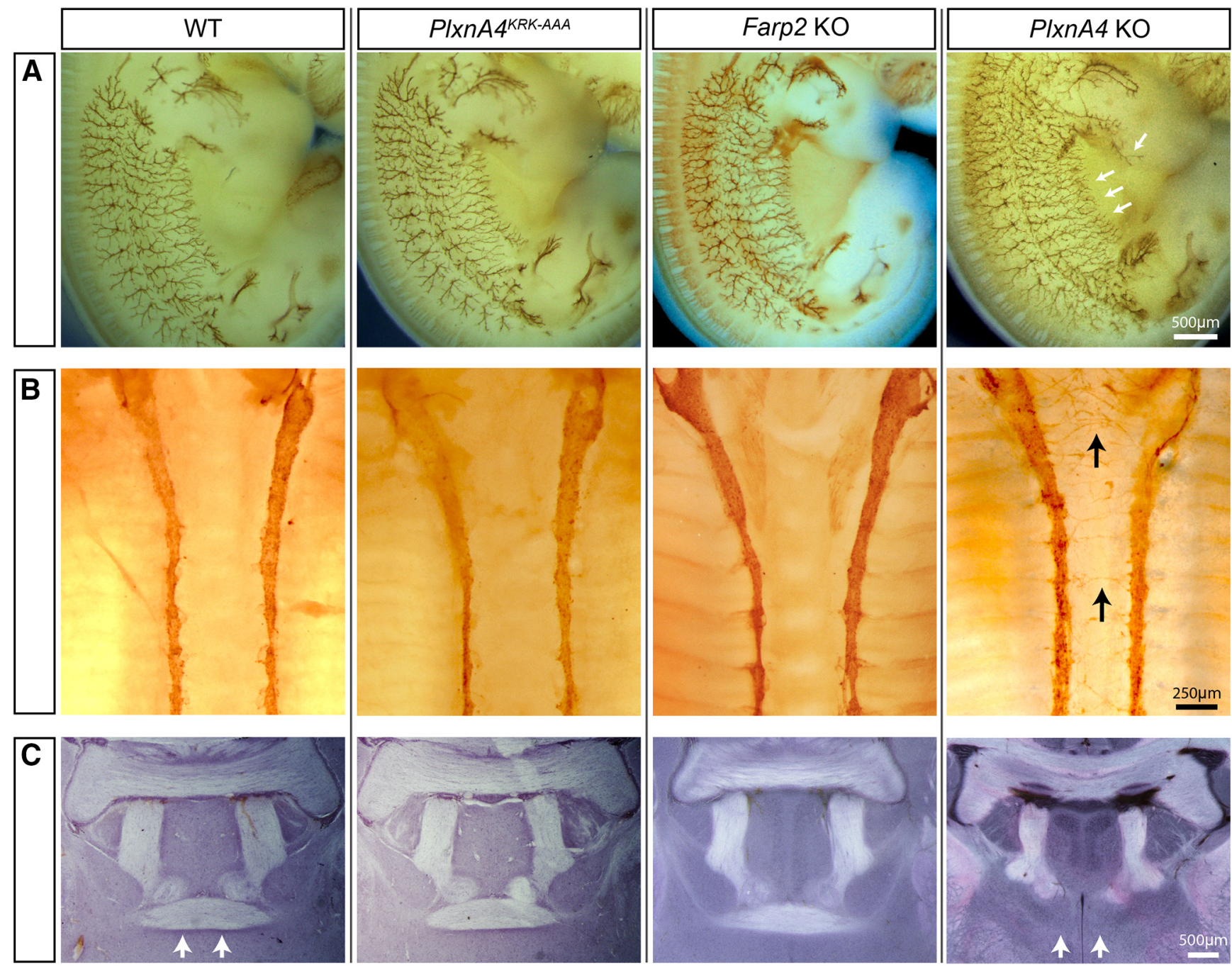

Figure 6. PlxnA $4^{K R K-A A A}$ and Farp2 KO animals show normal axonal projections in the PNS and CNS in vivo. A, WT, PlxnA4 ${ }^{K R K-A A A}$, Farp2 KO, or PlxnA4 KO E12.5 embryos were immunostained using a Neurofilament antibody to visualize cutaneous sensory axons pattern. PlXnA4 $4^{K R K-A A A}$ and Farp2 KO embryos were comparable with the WT, whereas the PlxnA4 K0 exhibited an expected hyperinnervation, including a previously described discrete phenotype (white arrows). Scale bar, $500 \mu \mathrm{m}$. B, WT, PlxnA ${ }^{\text {KRK-AAA }}$, Farp2 KO, or PlxnA4 K0 E13.5 embryos were immunostained using a TH antibody to visualize sympathetic axons. PlxnA4 ${ }^{K R K-A A A}$ and Farp2 KO embryos were comparable with the WT, whereas the PlxnA4 KO exhibited an expected medial protrusion of sympathetic axons (black arrows). Scale bar, $250 \mu \mathrm{m}$. C, WT, PlxnA4 ${ }^{K R K-A A A}$, Farp2 KO, or PlxnA4 KO P30 brains were stained using hematoxylin. PlxnA4 $4^{K R K-A A A}$ and Farp2 KO embryos were comparable with the WT, whereas the PIXnA4 KO exhibited an expected disrupted formation of the anterior commissure (white arrows). Scale bar, $500 \mu \mathrm{m}$.

WT (Fig. 5C-E; length: $p<0.0001, F=20.14$; DCI: $p=0.0005$, $F=13.81$; dendritic tips: $p<0.0001, F=21.94)$. To provide genetic evidence that FARP2 is indeed downstream of PlxnA4 signaling, we generated compound heterozygous animals, PlxnA4 $4^{K R K-A A A /+} ;$ Farp2 $^{+/-}$. The trans-heterozygous animals also exhibit reduced basal dendritic arbors, phenocopying the PlxnA4 KO (Fig. 5A-E; Sholl analysis between WT and PlxnA4 ${ }^{K R K-A A A /+} ;$ Farp2 $^{+/-}$and PlxnA4 KO; Sholl: $15 \mu \mathrm{m}$ : $p=0.0011, \quad F=15.93 ; 20 \mu \mathrm{m}: \quad p<0.0001, \quad F=38.17 ; \quad 25 \mu \mathrm{m}:$ $p=0.0002, F=25.58 ; 30 \mu \mathrm{m}: p=0.0004, F=20.52 ; 35 \mu \mathrm{m}: p=$ $0.0003, F=23.31 ; 40 \mu \mathrm{m}: p=0.0011, F=16.07 ; 45 \mu \mathrm{m}: p=0.0023$, $F=12.83 ; \quad 50 \mu \mathrm{m}: \quad p=0.0016, \quad F=14.43 ; \quad 55 \mu \mathrm{m}: \quad p=0.0002$, $F=25.78 ; 60 \mu \mathrm{m}: p=0.0015, F=14.63$; length: $p=0.0002 ; F=$ 25.71; DCI: $p=0.0005, F=19.45$; dendritic tips: $p=0.0003$, $F=23.90$ ). In addition, we compared the number of dendritic intersections, dendritic length, DCI, and number of dendritic tips between WT and PlxnA4 $4^{K R K-A A A /+}$ or Farp2 $2^{+/-}$single heterozygous animals. There was not a significant difference between WT and PlxnA4 $4^{K R K-A A A /+}$ or Farp $2^{+l-}$ heterozygous animals (images not shown); in contrast, there were significant differences between
$P l x n A 4^{+/ K R K-A A A}$ single heterozygous or Farp $2^{+/-}$single heterozygous and PlxnA4 $4^{K R K-A A A}$ mutant or PlxnA4 KO or Farp2 $\mathrm{KO}$ or compound heterozygous animals PlxnA ${ }^{\text {KRK-AAA/+ }} ;$ Farp $^{+/-}$ (compared with PlxnA4 $4^{+/ K R K-A A A}$ Sholl: $15 \mu \mathrm{m}: p=0.0156$, $F=4.601 ; 20 \mu \mathrm{m}: p=0.0002, F=13.32 ; 25 \mu \mathrm{m}: p<0.0001, F=18$; $30 \mu \mathrm{m}: p<0.0001, F=24.58 ; 35 \mu \mathrm{m}: p=0.0001, F=14.49 ; 40 \mu \mathrm{m}$ : $p=0.0013, \quad F=8.544 ; \quad 45 \mu \mathrm{m}: \quad p=0.0033, \quad F=6.923 ; \quad 50 \mu \mathrm{m}$ : $p=0.0027, F=7.251 ; 55 \mu \mathrm{m}: p=0.0009, F=9.292 ; 60 \mu \mathrm{m}: p=$ $0.0021, F=7.641$; length: $p<0.0001, F=20.18$; DCI: $p<0.0001$, $F=28.21$; dendritic tips: $p<0.0001, F=23.37$; compared with Farp $^{+-}$Sholl: $15 \mu \mathrm{m}: p=0.008, F=5.531 ; 20 \mu \mathrm{m}: p=0.0001$, $F=13.87 ; \quad 25 \mu \mathrm{m}: \quad p<0.0001, \quad F=15.45 ; \quad 30 \mu \mathrm{m}: \quad p<0.0001$, $F=18.43 ; 35 \mu \mathrm{m}: p=0.0004, F=10.96 ; 40 \mu \mathrm{m}: p=0.0039, \quad F=$ $6.625 ; 45 \mu \mathrm{m}: p=0.0065, \quad F=5.822 ; 50 \mu \mathrm{m}: p=0.0073$, $F=5.652 ; 55 \mu \mathrm{m}: p=0.0069, \quad F=5.748 ; 60 \mu \mathrm{m}: p=0.0289$; $F=3.819$; length: $p<0.0001, F=16.53$; DCI: $p<0.0001, F=$ 37.28; dendritic tips: $p<0.0001, F=20.32$ ).

While the Golgi staining is a great method for assessing overall neuronal morphologies, the impregnation of the silver nitrate is arbitrary for each neuron. Therefore, to confirm that indeed 

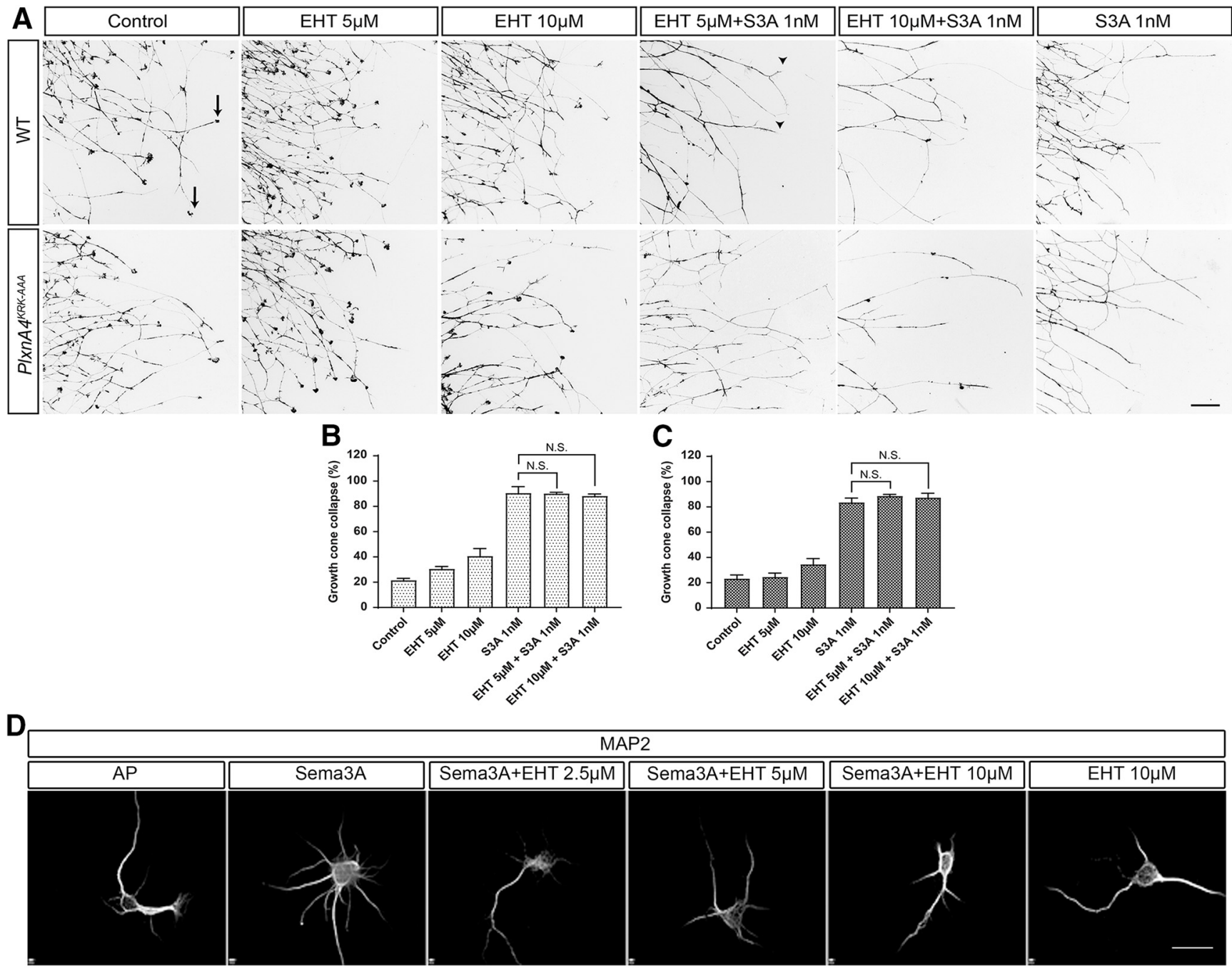

E

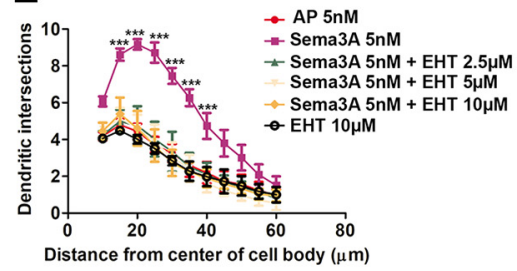

F

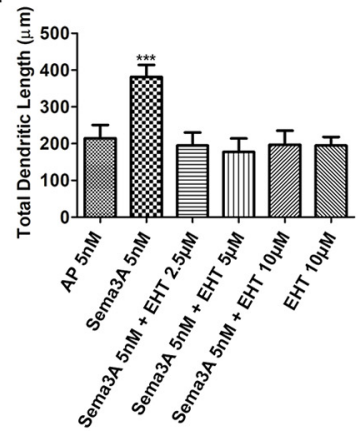

G

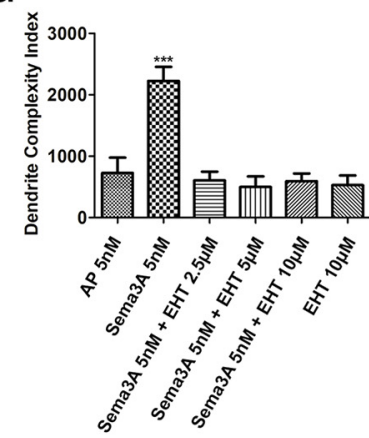

H

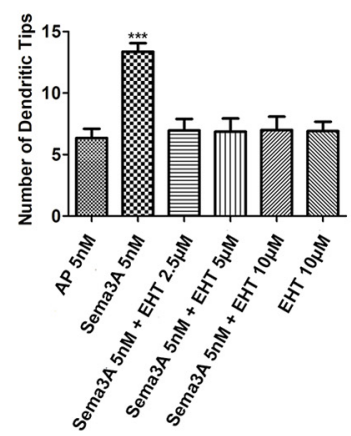

Figure 7. Inhibition of Rac signaling abolishes Sema3A-mediated cortical neuron dendrite elaboration but does not hinder Sema3A-dependent growth cone collapse of WT or PlxnA4 ${ }^{K R-A A A}$ DRG axons in vitro. A, DRG explants from WT and PIxnA4 $4^{K R K-A A A}$ littermates E13.5 embryos were grown for $48 \mathrm{~h}$, treated for 30 min with the pan-Rac inhibitor EHT 1864 at a concentration of 5 $\mu \mathrm{m}, 10 \mu \mathrm{m}$, or media alone as control. Then, $1 \mathrm{~nm}$ Sema3A or control conditioned media was added for 30 min, followed by fixation and phalloidin-rhodamine staining for assessment of growth cone collapse. Black arrows indicate intact growth cones. Arrowheads indicate collapsed growth cones. $\boldsymbol{B}, \boldsymbol{C}$, Quantification of collapse response as a mean percentage of collapsed growth cones out of the total \pm SEM in WT and PIxnA4 ${ }^{K R K-A A A}$ axons, respectively; N.S., Non Significant. Scale bar, $50 \mu \mathrm{m}$. $\boldsymbol{D}$, Representative confocal micrographs of dissociated primary cortical neurons obtained from WT E13.5 embryos. The neurons were treated with 5 nm AP, 5 nм Sema3A, 5 nm Sema3A $+2.5 \mu$ m EHT, 5 nm Sema3A $+5 \mu$ m EHT, and 5 nm Sema3A $+10 \mu$ m EHT. $\boldsymbol{E}-\boldsymbol{H}$, Sholl analysis of dendritic intersections $(\boldsymbol{E})$, total dendritic length $(\boldsymbol{F})$, the $D C l(\boldsymbol{G})$, and number of dendritic tips $(\boldsymbol{H})$ for all treatment conditions described above. Data are mean \pm SEM from three independent cultures. ${ }^{* *} p<0.001$ (two-way ANOVA with post hoc Tukey test). Scale bars: $\boldsymbol{A}, \boldsymbol{D}, 25 \mu \mathrm{m}$.

layer 5 neuron basal dendrites are exhibiting the reduced dendritic arbor phenotype observed, we crossed the PlxnA4 ${ }^{K R K-A A A}$ mutant or the Farp2 KO with Thy1-EGFP reporter mice to genetically label layer 5 neurons. We used the M line of Thy1EGFP to sparsely label specific layer 5 neurons for optimal visualization of the entire dendritic arbor of each neuron. Consistent with our Golgi staining analysis, we found that $P l x n A 4^{K R K-A A A}$ mutant;Thy1-EGFP ${ }^{+}$and PlxnA4 ${ }^{\mathrm{KRK}-A A A /+} ; \mathrm{Farp}^{+/-}$;Thy1$\mathrm{EGFP}^{+}$(trans-heterozygous) neurons displayed significantly reduced basal dendritic arbors compared with WT Thyl-EGFP ${ }^{+}$ neurons (Fig. 5F,G; Sholl: $15 \mu \mathrm{m}: p<0.0025, F=13.94 ; 20 \mu \mathrm{m}$ : $p<0.0076, \quad F=9.531 ; 25 \mu \mathrm{m}: \quad p<0.0031, \quad F=12.99 ; \quad 30 \mu \mathrm{m}$ : 


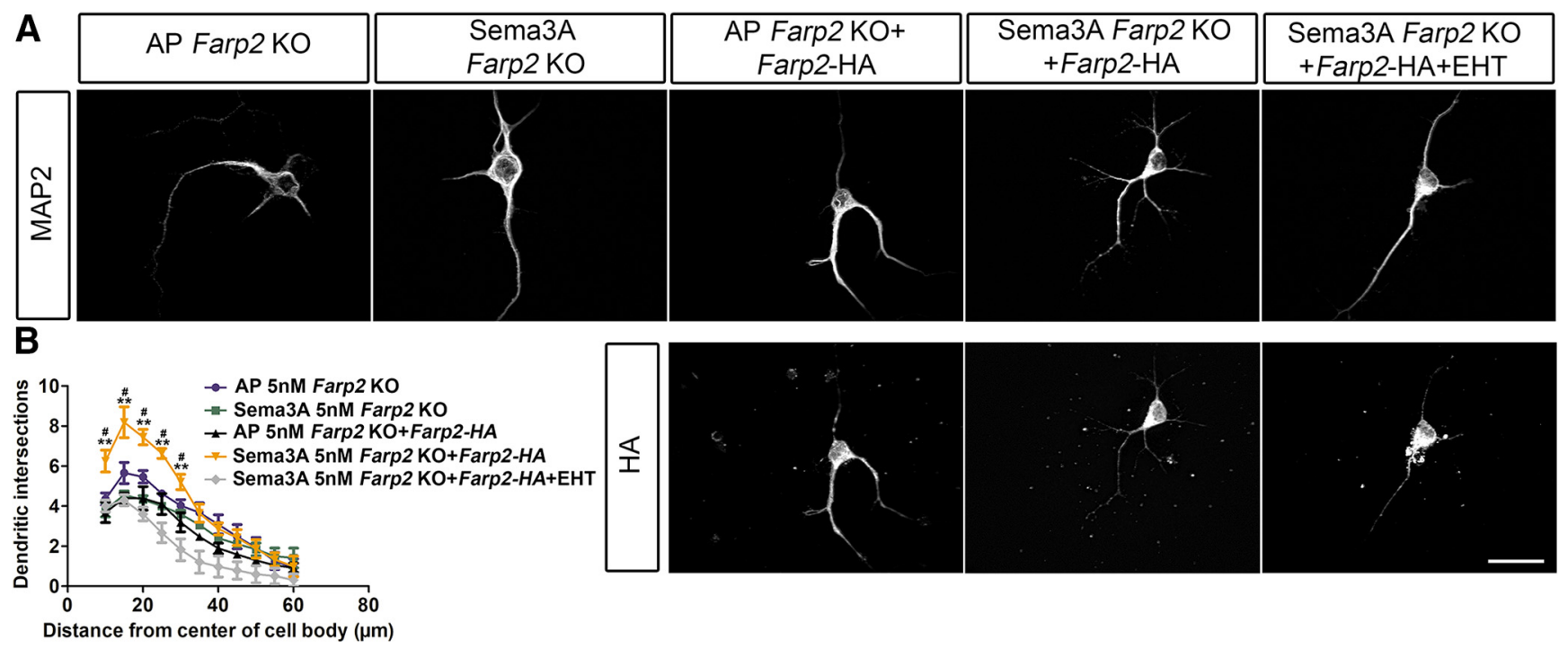

Figure 8. Sema3A-dependent dendritic arborization relies on the FARP2 GEF and downstream Rac1 GTPase activation. $A$, Representative confocal micrographs of dissociated primary neurons

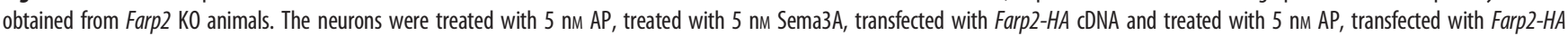
CDNA and treated with $5 \mathrm{~nm}$ Sema3A and transfected with Farp2-HA cDNA, treated with $5 \mathrm{~nm}$ Sema3A $+2.5 \mathrm{uM}$ EHT for $24 \mathrm{~h}$. B. Sholl analysis of dendritic intersections in the five different groups. Data are mean \pm SEM from three independent cultures. ${ }^{* *},{ }^{*} p<0.01$ (two-way ANOVA with post hoc Tukey test). ${ }^{*}$ Statistical difference between Farp2 K0+Farp2-HA and Farp2 KO + Farp2- HA+Sema3A. "Statistical difference between Farp2 KO+Farp2-HA+Sema3A and Farp2 KO+Farp2-HA+Sema3A + EHT. Scale bars: A, $25 \mu \mathrm{m}$.

$p<0.0006, F=21.14 ; 35 \mu \mathrm{m}: p<0.0007, F=20.72 ; 40 \mu \mathrm{m}: p=$ $0.0045, F=11.44 ; 45 \mu \mathrm{m}: p=0.0177, F=6.968)$.

In contrast to dendritic development, both $P l x n A 4^{\text {KRK-AAA }}$ mutant and Farp2 KO embryos at E12.5 showed normal sensory cutaneous axon patterning in the PNS, comparable with the WT, as opposed to the defasciculated, highly branched, and misguided axonal projections seen in the PlxnA4 null (Fig. 6A, arrows). Furthermore, it is well established that Sema3A-PlxnA4 signaling can exert axonal repulsion on different neuronal populations in both the PNS and CNS (Gu et al., 2003; Huber et al., 2005; Yaron et al., 2005). In addition to repelling peripheral DRG axons, PlxnA4 is also required in the PNS for the proper projections of sympathetic axons, and for the formation of the anterior commissure in the CNS (Yaron et al., 2005; Waimey et al., 2008). Therefore, we asked whether the KRK motif in the PlxnA4 receptor is dispensable only for PNS DRG axon repulsion or can this be a general principal for Sema3A signaling in axon guidance. TH staining of the sympathetic ganglia showed normal axonal patterns in PlxnA4 ${ }^{K R K-A A A}$ mutant and Farp2 KO E13.5 embryos similar to WT control, whereas PlxnA4 KO animals displayed disorganized axonal projections toward the midline (Fig. 6B). Hematoxylin staining of coronal sections from WT, PlxnA4 ${ }^{\text {KRK-AAA }}$, PlxnA4 KO, and Farp2 KO brains at $\mathrm{P} 30$ showed normal anterior commissure projections in both PlxnA4 ${ }^{\text {KRK-AAA }}$ mutant and Farp $2 \mathrm{KO}$ animals, similar to the WT, but in contrast to the missing commissure in the PlxnA4 KO (Fig. $6 C)$.

Together, these results suggest that the PlxnA4 ${ }^{\mathrm{KRK}}$-FARP2 signaling pathway is necessary to promote layer 5 pyramidal neuron basal dendrite elaboration but is dispensable for axonal repulsion in vitro and in vivo.

Inhibition of Rac activity perturbs Sema3A-mediated cortical neuron dendrite elaboration, but not axon growth cone collapse

One of the major targets of FARP2 GEF is the small GTPase Rac1, but not other members of the Rho GTPase family: CDC42 or RhoA (Kubo et al., 2002; Toyofuku et al., 2005; He et al.,
2013). A previous study showed that overexpression of FARP2 in murine primary neurons induced shortened neurites (Kubo et al., 2002); however, the physiological importance of FARP2-Rac1 signaling pathway in the nervous system is not clear. Therefore, we first asked whether Rac activation is required downstream of Sema3A-signaling for axonal growth cone collapse and dendrite morphogenesis using a pharmacological approach. We used a validated pan-Rac GTPase inhibitor, EHT1864 (Shutes et al., 2007; Onesto et al., 2008), in a dosage-dependent manner on cultured WT DRG neurons and found that this inhibitor had no effect on Sema3A-induced growth cone collapse (Fig. 7A-C). However, WT-dissociated cortical neurons treated with the Rac inhibitor for $2 \mathrm{~h}$ before Sema3A stimulation, at concentrations of $2.5,5$, or $10 \mu \mathrm{M}$, showed complete inhibition of dendritic elaboration, compared with Sema3A treated neurons without the inhibitor. In contrast, there were no significant differences in dendritic morphology between AP-treated WT neurons versus AP-treated + EHT at different concentrations $(2.5,5$, and $10 \mu \mathrm{M})$ (Fig. 7D). Quantifications of dendritic intersections by Sholl analysis (Fig. 7E), total dendritic length (Fig. 7F), DCI (Fig. 7G), and average number of dendritic tips (Fig. $7 \mathrm{H}$ ) showed significant differences between WT neurons treated with Sema3A only compared with Sema3A $+2.5 \mu \mathrm{M}$ EHT, Sema3A $+5 \mu \mathrm{M}$ EHT, Sema3A $+10 \mu \mathrm{M}$ EHT, 2.5, 5, or $10 \mu \mathrm{M}$ EHT alone or control (no Sema3A, no EHT) (two-way ANOVA, Sholl: $15 \mu \mathrm{m}$ : $p=0.0019, \quad F=7.822 ; \quad 20 \mu \mathrm{m}: \quad p=0.0012, \quad F=8.652 ; \quad 25 \mu \mathrm{m}$ : $p=0.0005, F=10.19,30 \mu \mathrm{m}: p=0.0020, F=7.798 .117 ; 35 \mu \mathrm{m}: p=$ $0.0019, F=7.895 ; 40 \mu \mathrm{m}: p=0.0181, F=4.492$; length: $p=0.0276$, $F=3.674$; DCI: $p=0.0030, F=9.454$; and dendritic tips: $p=$ $0.0013, F=7.381)$. These results establish that Rac activation is required for Sema3A-mediated dendrite outgrowth and branching.

\section{Sema3A-mediated cortical neuron dendrite elaboration requires Rac1 activation by FARP2 GEF}

To support our hypothesis that Sema3A-mediated dendrite elaboration involves a FARP2-Rac signaling pathway, we used Farp2 $\mathrm{KO}$ cortical neurons, which we showed are insensitive to 
A

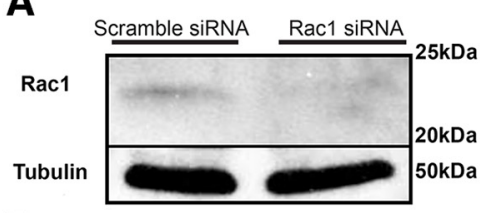

B
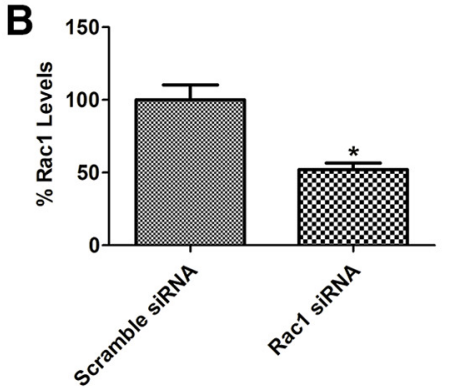

C
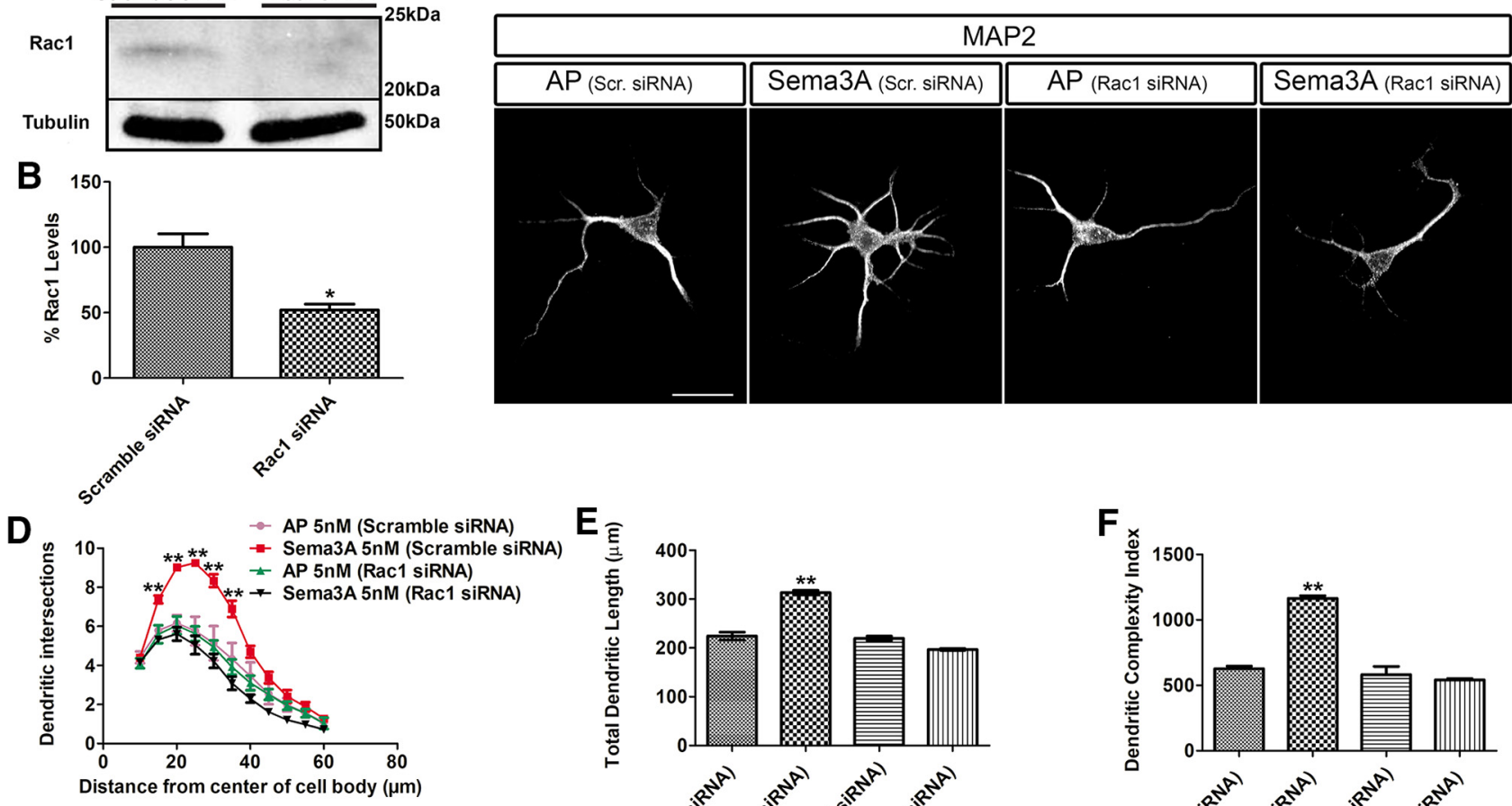

$\mathbf{E}_{\overline{\mathrm{E}}}$
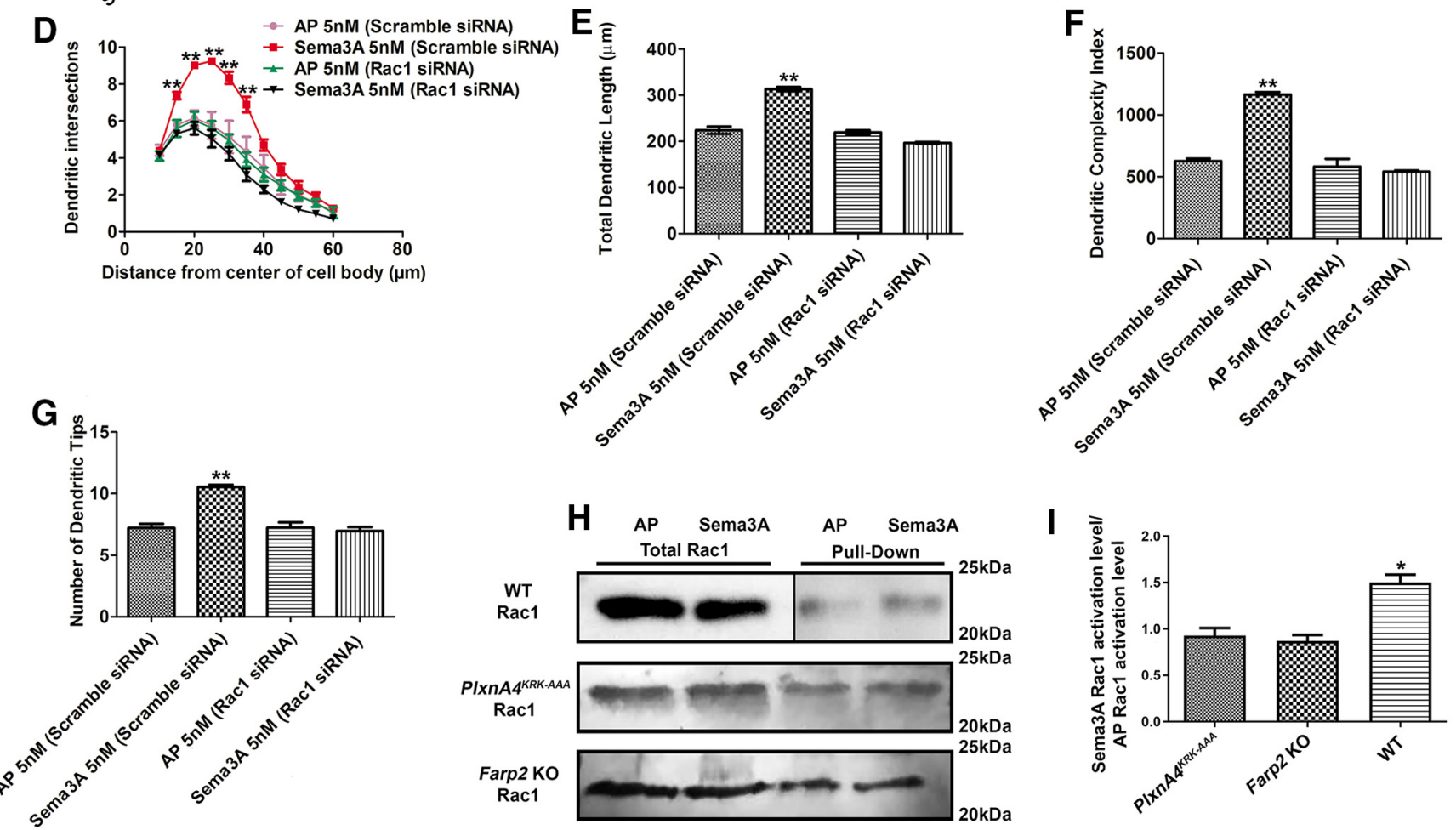

Figure 9. Sema3A increases Rac activation in cortical neurons and requires the presence of Rac1 for dendrite elaboration. $\boldsymbol{A}$, Rac1-specific siRNA decrease Rac1 total protein levels. $\boldsymbol{B}$, Quantification of Rac1 levels from three independent experiments. ${ }^{*} p<0.05$ (Student's $t$ test). C, Representative confocal micrographs of dissociated primary cortical neurons obtained from WT E13.5 embryos transfected either with scramble siRNA or Rac1-specific siRNA. $\boldsymbol{D}$, Sholl analysis of dendritic intersections. $\boldsymbol{E}$, Total dendritic length. $\boldsymbol{F}$, DCI. $\boldsymbol{G}$, Number of dendritic tips for all treatment conditions described above. Data are mean \pm SEM from three independent cultures. ${ }^{* *} p<0.01$ (two-way ANOVA with post hoc Tukey test). Scale bars: $\mathbf{C}, 25 \mu \mathrm{m}$. $\boldsymbol{H}$, Pulldown assay showing the effect of 30 min stimulation of Sema3A on Rac1 activation in WT neurons, in PlxnA4 ${ }^{\text {KRK-AAA }}$, and in Farp2 KO neurons. I, Quantification of Rac1-GTP fold change from three independent experiments. ${ }^{*} p<0.05$ (one-way ANOVA).

Sema3A-induced dendritic elaboration (Fig. 3). First, we demonstrate that by overexpressing Farp2 cDNA into Farp2 KO neurons, the sensitivity to Sema3A-induced dendritic elaboration is restored compared with Farp2 KO neurons transfected with Farp2 cDNA without Sema3A treatment (Fig. $8 A, B$; two-way ANOVA, Sholl: $10 \mu \mathrm{m}: p=0.0081, F=12.24 ; 15 \mu \mathrm{m}: p=0.0011$, $F=24.47 ; \quad 20 \mu \mathrm{m}: \quad p=0.0007, \quad F=27.95 ; \quad 25 \mu \mathrm{m}: \quad p=0.0008$, $F=26.81,30 \mu \mathrm{m}: p=0.0086, F=11.93)$. However, Farp2 KO neurons were unable to elaborate dendrites when transfected with Farp2 cDNA $+2.5 \mu \mathrm{M}$ EHT and treated with Sema3A. Sholl analysis showed that the number of dendritic intersections significantly decreased between 10 and $30 \mu \mathrm{m}$ away from the cell soma in Farp2 KO neurons rescued with Farp2 cDNA and treated with Sema3A + EHT compared with Farp2 KO rescued with FARP2 cDNA and treated with Sema3A only (Fig. $8 A, B$; two-way ANOVA, Sholl: $10 \mu \mathrm{m}: p=0.0132, F=10.05 ; 15 \mu \mathrm{m}$ : $p=0.0015, F=22.08 ; 20 \mu \mathrm{m}: p=0.0023, F=19.24 ; 25 \mu \mathrm{m}: p=$ $0.0021, F=19.84,30 \mu \mathrm{m}: p=0.0021, F=19.87)$.

While the results from our pharmacological experiments are consistent with Rac1 as the downstream target of FARP2, EHT inhibits all Rac activity (Shutes et al., 2007; Onesto et al., 2008) activity. Using siRNA specific to Racl, we found that WT cortical neurons transfected with Racl siRNA not only expressed a significantly lower level of Rac1 (Fig. 9A,B; Student's $t$ test, $t=4.245, p=0.0132$ ), but were also nonresponsive to Sema3Ainduced dendrite outgrowth and branching compared with the Sema3A-scramble siRNA (Fig. 9C-G; two-way ANOVA, Sholl: $15 \mu \mathrm{m}: p=0.0111, F=10.81 ; 20 \mu \mathrm{m}: p=0.0032, F=17.16 ; 25 \mu \mathrm{m}$ : 

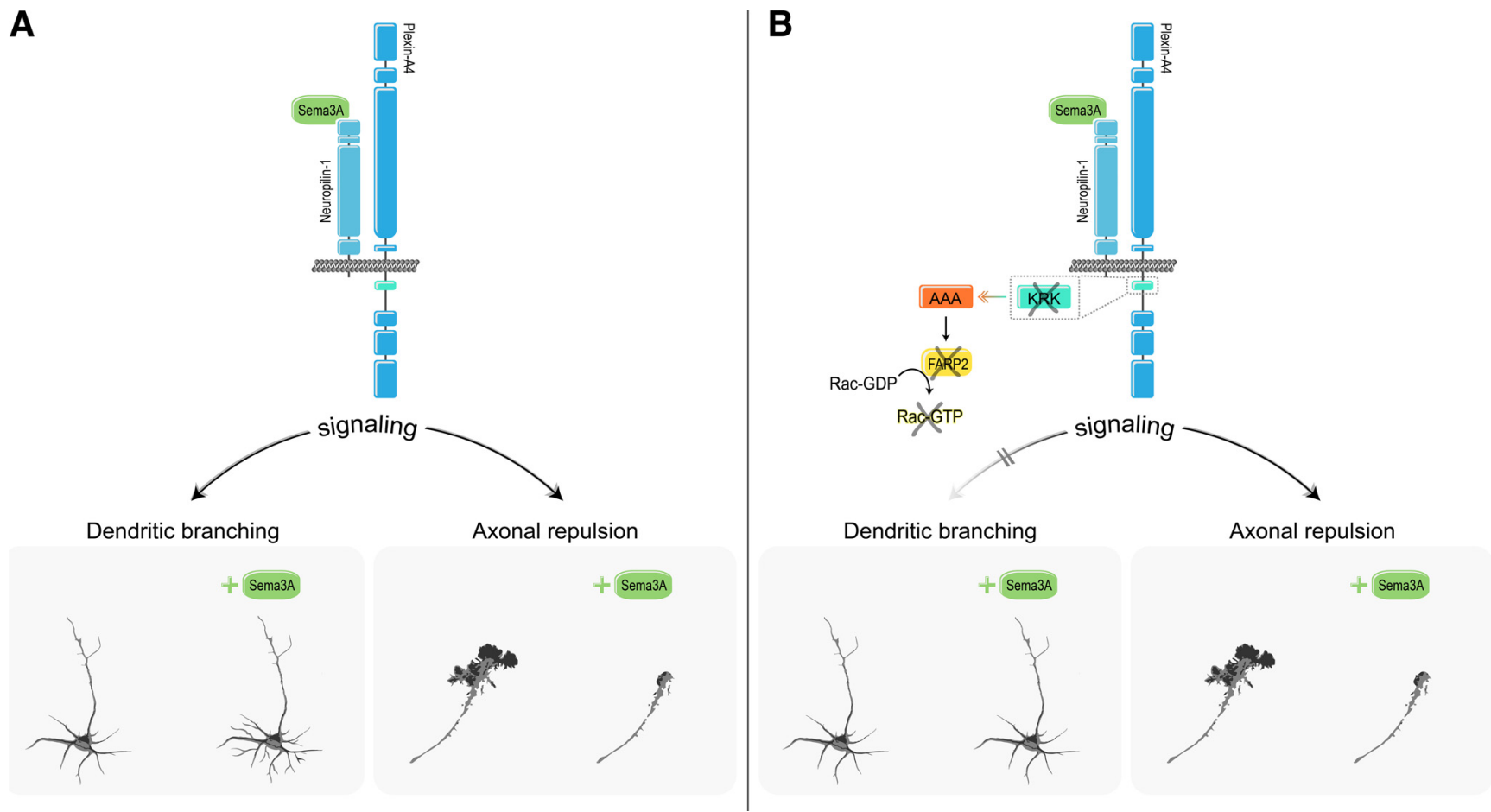

Figure 10. Differential requirement for the KRK motif and its downstream signaling effector FARP2 in Plexin-A4-mediated cellular responses. $A$, Sema3A-Nrp1/Plexin-A4 signaling promotes basal dendrite elaboration in deep-layer pyramidal cortical neurons on one hand and growth cone collapse and axonal repulsion on the other. B, Substitution of the KRK motif of Plexin-A4 to AAA, ablation of the Plexin-A4-binding effector, the Rac1 GEF FARP2, or inhibition of Rac1-specifically abrogate dendrite elaboration but not growth cone collapse and axonal repulsion.

$p=0.0026, F=18.48 ; 30 \mu \mathrm{m}: p=0.0047, F=15.08 ; 35 \mu \mathrm{m}: p=$ $0.0055, F=14.14$; length: $p<0.0001, F=112.6$; DCI: $p<0.0001$, $F=70.5$; dendritic tips: $p=0.0006, F=29.49)$. Finally, we asked whether Racl activity is altered following Sema3A signaling in primary cortical neurons. Using a biochemical assay to pull down the downstream target of Rac1, PAK1, we quantified the level of active Racl in its GTP bound state (for details, see Materials and Methods). We found that the level of Rac1-GTP increased in WT primary cortical neurons following $30 \mathrm{~min}$ treatment with AP-tagged Sema3A compared with the vehicle control AP treatment (Student's $t$ test $t=3.168, p=0.0339$ ). Moreover, we found that Sema3A treatment on PlxnA4 ${ }^{\text {KRK-AAA }}$ and on Farp $2 \mathrm{KO}$ cortical neurons did not induce any significant increase in Racl activity (Fig. 9H,I, one-way ANOVA $p=0.0022$; $F=20.14$ ). Together, our results demonstrate that Sema3Ainduced cortical neuron dendrite elaboration requires the activation of the PlxnA4 ${ }^{\mathrm{KRK}}$ motif, which signals through FARP2 GEF to activate Rac1.

\section{Discussion}

How a finite number of molecular cues can sculpt the precise neuronal connections of complex circuits and induce diverse cellular responses is a fundamental question in neural development. Here, we show that Sema3A signals through the PlxnA4 receptor by selectively engaging the cytoplasmic KRK motif to specifically promote cortical neuron basal dendrite elaboration, but not CNS or PNS axon guidance. Although we cannot completely rule out the involvement of another coreceptor, which interacts with PlxnA4 in a KRK-dependent manner, currently there is no evidence supporting this notion. Our work supports the hypothesis that this KRK sequence is a signaling motif. First, FARP2 interacts with PlxnA4 in a KRK-dependent manner (Mlechkovich et al., 2014). Second, PlxnA4 ${ }^{K R K-A A A}$ and Farp2 interact genetically in vivo. Additionally, this PlxnA4-FARP2 pathway requires the downstream target of FARP2, the small GTPase Rac1, to be active in cortical neurons but not in DRG sensory axons. Together, our results uncovered, for the first time, the selective Sema3A pathway through the KRK motif of the PlxnA4 receptor that activates FARP2-Rac1 signaling to control dendrite morphogenesis specifically in layer 5 cortical neurons (Fig. 10). In agreement with our work, a recent study suggested a functional divergence of the PlexinB motifs in the Drosophila olfactory circuit (Guajardo et al., 2019). Unlike our study, the downstream elements to these PlexinB motifs remain elusive.

\section{PlxnA4-FARP2-Rac1 pathway is required for dendrite morphogenesis but not for axon guidance}

Our in vitro and in vivo analyses of the PlxnA4 $4^{\text {KRK-AAA }}$ mouse agree with our previous overexpression studies of PlxnA4 mutants in cultured neurons (Mlechkovich et al., 2014). While our previous study showed that Sema3A-mediated axon growth cone collapse is disrupted by Farp2 siRNA knockdown in DRG neurons, our current results using the Farp2 KO mouse contradict the in vitro knockdown findings. The basis for this difference may be due to off-target effects of the siRNA or compensatory mechanisms in the Farp2 KO DRGs. Another study argued that Sema3A signaling mediates the dissociation of FARP2 from PlexinA1, resulting in the activation of Rac1 by FARP2 in axon repulsion assays of chick DRG neurons (Toyofuku et al., 2005). We have not found any evidence that this pathway is required for DRG axonal repulsion in vitro or for any repulsive guidance events of different neuronal types in vivo. Although there might be species differences between mice and chicken in Sema3A downstream signaling that may account for these discrepancies, our data support the model that this pathway is exclusively required for dendrite morphogenesis (Fig. 10). 
While previous studies showed that Sema3A could increase Racl activity, they were mainly performed with either COS7 or HEK 293 cells (Turner et al., 2004; Toyofuku et al., 2005). We showed, for the first time, that Sema3A signaling in cortical neurons increases Racl activity. Another study using Racl siRNA in nonstimulated hippocampal neurons showed impairment in dendrite but not axon development (Gualdoni et al., 2007). In contrast, our data suggest that, in cortical neurons, Racl is needed for Sema3A-induced dendritic elaboration but not in the basal state. Furthermore, we corroborated our knockdown findings by inhibiting Rac1 activity with a pan-Rac inhibitor (EHT1864), which only perturbed Sema3A-mediated dendritic elaboration but not axonal growth cone collapse. While our results cannot rule out the contribution of other Rac isoforms (Rac2, Rac3, and RhoG), there are several reasons to support Rac1 as the favorite candidate. First, Rac2 expression has been shown to be restricted to hematopoietic cells (Roberts et al., 1999). Second, it has been proposed that Rac1 and Rac3 have opposing roles. While Rac1 is localized to the plasma membrane and cell protrusions, required for cell differentiation, neurite outgrowth, and involved in dendrites and spines development and maintenance (Luo, 2000; Gonzalez-Billault et al., 2012), Rac3 is localized in the perinuclear region, required for maintaining an undifferentiated state, and interferes with integrin-mediated cellmatrix adhesions (Corbetta et al., 2005; Hajdo-Milasinovic et al., 2007, 2009; Waters et al., 2008). The least studied is RhoG, which is expressed broadly (de Curtis, 2008) and previously shown to reduce dendrite complexity in hippocampal neurons (Franke et al., 2012; Schulz et al., 2016), rather than promoting dendritic arborization, as in our Racl results. Together, both in vitro and in vivo results support PlxnA4 ${ }^{\mathrm{KRK}}{ }_{\text {-FARP2-Rac1 as the main }}$ pathway in Sema3A-mediated signaling in cortical neuron dendrite morphogenesis.

How is the specificity of the PlxnA4-FARP2-Racl pathway in cortical neuron dendritic morphogenesis achieved? One simple explanation is differential gene expression. However, all the components of this pathway are expressed both in cortical and DRG neurons during development (Mlechkovich et al., 2014). A recent single-cell analysis of DRG neurons at different developmental stages also detected the expression of FARP2, although at low levels, and expression of Racl (Sharma et al., 2020). Another possibility is that the KRK motif is inaccessible to FARP2 in DRG neurons. While beyond the scope of this study, this may be achieved by another FERM domain-containing protein, which is not required for signaling, or by differential lipid composition of the axons and dendrite membranes, as the KRK motif is located adjacent to the transmembrane domain.

\section{Sema3A signaling as a mechanism for promoting dendrite morphogenesis and remodeling in CNS neurons}

Previous studies have identified the Src kinases (SFKs) as downstream effectors of Sema3A signaling in cortical basal dendrite elaboration (Sasaki et al., 2002; Morita et al., 2006). Recently, the protein tyrosine phosphatase $\delta(\operatorname{Ptp} \delta)$ was shown to interact with Nrp1 and to regulate the activity of SFKs in cortical neurons (Nakamura et al., 2017). However, these studies also demonstrated that the SFKs and $\operatorname{Ptp} \delta$ control the growth cone collapse response mediated by Sema3A (Sasaki et al., 2002; Nakamura et al., 2017). Therefore, the SFKs do not confer functional specificity, but rather they may be considered as nonspecific signaling elements that are required for any type of Sema3A function.

While Sema3A can promote dendrite branching in dissociated hippocampal neurons (Fenstermaker et al., 2004), it also regulates the polarization of axon versus dendrite during hippocampal development (Shelly et al., 2011). Exogenous application of Sema3A suppressed undifferentiated neurites from differentiating into axons and promoted dendrite growth by elevating and reducing cGMP and cAMP levels, respectively. The ability of Sema3A to induce dendrite differentiation versus axon formation is recapitulated in Xenopus spinal neurons (Nishiyama et al., 2011). While the mechanism is unclear, Sema3A treatment of Xenopus spinal interneurons induced dendrite identity specification by simultaneous suppression of axon identity through regulation of cGMP signaling and voltage-sensitive $\mathrm{Ca}^{2+}$ channel $\mathrm{Ca}_{\mathrm{V}} 2.3$ expression. Activity-dependent Sema3A-induced dendrite branching is an interesting notion. Using an overexpression paradigm with tagged FARP1, Nrp1, and PlxnA1, another study showed the other member of the FERM domain containing RhoGEF FARP1 can associate with Nrp1/PlxnA1 complexes in HEK293 cells (Cheadle and Biederer, 2014). While Sema3A treatment of 21 DIV hippocampal neurons was unable to induce dendrite branching when FARP1 was knocked down in the presence of TTX, overexpression of PlxnA1 with TTX resulted in increased length of total dendritic branches, suggesting that the Semaphorin signaling pathway in hippocampal neurons is different from that in cortical neurons, which we show to be Sema3ANrp1/PlxnA4-FARP2-Rac1.

In conclusion, our study uncovers PlxnA4 ${ }^{\mathrm{KRK}} / \mathrm{FARP} 2 / \mathrm{Rac} 1$ as a selective signaling pathway toward cortical pyramidal dendritic elaboration. Broadly, our results shed light on a longstanding question regarding the ability of multifunctional receptors to trigger diverse outputs, by demonstrating how distinct morphologic changes can be pinpointed to specific receptor signaling motifs and their downstream effectors.

\section{References}

Anzo M, Sekine S, Makihara S, Chao K, Miura M, Chihara T (2017) Dendritic Eph organizes dendrodendritic segregation in discrete olfactory map formation in Drosophila. Genes Dev 31:1054-1065.

Barnes AP, Polleux F (2009) Establishment of axon-dendrite polarity in developing neurons. Annu Rev Neurosci 32:347-381.

Bradford MM (1976) A rapid and sensitive method for the quantitation of microgram quantities of protein utilizing the principle of protein-dye binding. Anal Biochem 72:248-254.

Castellani V, Chedotal A, Schachner M, Faivre-Sarrailh C, Rougon G (2000) Analysis of the L1-deficient mouse phenotype reveals cross-talk between Sema3A and L1 signaling pathways in axonal guidance. Neuron 27:237249.

Chari R, Mali P, Moosburner M, Church GM (2015) Unraveling CRISPRCas9 genome engineering parameters via a library-on-library approach. Nat Methods 12:823-826.

Chauvet S, Cohen S, Yoshida Y, Fekrane L, Livet J, Gayet O, Segu L, Buhot MC, Jessell TM, Henderson CE, Mann F (2007) Gating of Sema3E/ PlexinD1 signaling by neuropilin-1 switches axonal repulsion to attraction during brain development. Neuron 56:807-822.

Cheadle L, Biederer T (2014) Activity-dependent regulation of dendritic complexity by semaphorin 3A through Farp1. J Neurosci 34:7999-8009.

Corbetta S, Gualdoni S, Albertinazzi C, Paris S, Croci L, Consalez GG, de Curtis I (2005) Generation and characterization of Rac3 knockout mice. Mol Cell Biol 25:5763-5776.

de Curtis I (2008) Functions of Rac GTPases during neuronal development. Dev Neurosci 30:47-58.

Doench JG, Fusi N, Sullender M, Hegde M, Vaimberg EW, Donovan KF, Smith I, Tothova Z, Wilen C, Orchard R, Virgin HW, Listgarten J, Root DE (2016) Optimized sgRNA design to maximize activity and minimize off-target effects of CRISPR-Cas9. Nat Biotechnol 34:184-191.

Doench JG, Hartenian E, Graham DB, Tothova Z, Hegde M, Smith I, Sullender M, Ebert BL, Xavier RJ, Root DE (2014) Rational design of highly active sgRNAs for CRISPR-Cas9-mediated gene inactivation. Nat Biotechnol 32:1262-1267. 
Fenstermaker V, Chen Y, Ghosh A, Yuste R (2004) Regulation of dendritic length and branching by semaphorin 3A. J Neurobiol 58:403412 .

Ferreira TA, Blackman AV, Oyrer J, Jayabal S, Chung AJ, Watt AJ, Sjostrom PJ, van Meyel DJ (2014) Neuronal morphometry directly from bitmap images. Nat Methods 11:982-984.

Franke K, Otto W, Johannes S, Baumgart J, Nitsch R, Schumacher S (2012) miR-124-regulated RhoG reduces neuronal process complexity via ELMO/Dock180/Rac1 and Cdc42 signalling. EMBO J 31:2908-2921.

Gaitanos TN, Koerner J, Klein R (2016) Tiam-Rac signaling mediates transendocytosis of ephrin receptor EphB2 and is important for cell repulsion. J Cell Biol 214:735-752.

Gonzalez-Billault C, Munoz-Llancao P, Henriquez DR, Wojnacki J, Conde C, Caceres A (2012) The role of small GTPases in neuronal morphogenesis and polarity. Cytoskeleton (Hoboken) 69:464-485.

Gu C, Rodriguez ER, Reimert DV, Shu T, Fritzsch B, Richards LJ, Kolodkin AL, Ginty DD (2003) Neuropilin-1 conveys semaphorin and VEGF signaling during neural and cardiovascular development. Dev Cell 5:45-57.

Guajardo R, Luginbuhl DJ, Han S, Luo L, Li J (2019) Functional divergence of Plexin B structural motifs in distinct steps of Drosophila olfactory circuit assembly. eLife 8:e48594.

Gualdoni S, Albertinazzi C, Corbetta S, Valtorta F, de Curtis I (2007) Normal levels of Rac1 are important for dendritic but not axonal development in hippocampal neurons. Biol Cell 99:455-464.

Hajdo-Milasinovic A, Ellenbroek SI, van Es S, van der Vaart B, Collard JG (2007) Rac1 and Rac3 have opposing functions in cell adhesion and differentiation of neuronal cells. J Cell Sci 120:555-566.

Hajdo-Milasinovic A, van der Kammen RA, Moneva Z, Collard JG (2009) Rac3 inhibits adhesion and differentiation of neuronal cells by modifying GIT1 downstream signaling. J Cell Sci 122:2127-2136.

He X, Kuo YC, Rosche TJ, Zhang X (2013) Structural basis for autoinhibition of the guanine nucleotide exchange factor FARP2. Structure 21:355-364.

Hsu PD, Scott DA, Weinstein JA, Ran FA, Konermann S, Agarwala V, Li Y, Fine EJ, Wu X, Shalem O, Cradick TJ, Marraffini LA, Bao G, Zhang F (2013) DNA targeting specificity of RNA-guided Cas9 nucleases. Nat Biotechnol 31:827-832.

Huber AB, Kolodkin AL, Ginty DD, Cloutier JF (2003) Signaling at the growth cone: ligand-receptor complexes and the control of axon growth and guidance. Annu Rev Neurosci 26:509-563.

Huber AB, Kania A, Tran TS, Gu C, De Marco Garcia N, Lieberam I, Johnson D, Jessell TM, Ginty DD, Kolodkin AL (2005) Distinct roles for secreted semaphorin signaling in spinal motor axon guidance. Neuron 48:949-964.

Jan YN, Jan LY (2010) Branching out: mechanisms of dendritic arborization. Nat Rev Neurosci 11:316-328.

Jin Z, Strittmatter SM (1997) Rac1 mediates collapsin-1-induced growth cone collapse. J Neurosci 17:6256-6263.

Kantor DB, Chivatakarn O, Peer KL, Oster SF, Inatani M, Hansen MJ, Flanagan JG, Yamaguchi Y, Sretavan DW, Giger RJ, Kolodkin AL (2004) Semaphorin 5A is a bifunctional axon guidance cue regulated by heparan and chondroitin sulfate proteoglycans. Neuron 44:961-975.

Kolodkin AL, Tessier-Lavigne M (2011) Mechanisms and molecules of neuronal wiring: a primer. Cold Spring Harb Perspect Biol 3:a001727.

Kubo T, Yamashita T, Yamaguchi A, Sumimoto H, Hosokawa K, Tohyama M (2002) A novel FERM domain including guanine nucleotide exchange factor is involved in Rac signaling and regulates neurite remodeling. J Neurosci 22:8504-8513.

Lom B, Cohen-Cory S (1999) Brain-derived neurotrophic factor differentially regulates retinal ganglion cell dendritic and axonal arborization in vivo. J Neurosci 19:9928-9938.

Luo L (2000) Rho GTPases in neuronal morphogenesis. Nat Rev Neurosci 1:173-180.

Mlechkovich G, Peng SS, Shacham V, Martinez E, Gokhman I, Minis A, Tran TS, Yaron A (2014) Distinct cytoplasmic domains in Plexin-A4 mediate diverse responses to semaphorin $3 \mathrm{~A}$ in developing mammalian neurons. Sci Signal 7:ra24.

Morita A, Yamashita N, Sasaki Y, Uchida Y, Nakajima O, Nakamura F, Yagi T, Taniguchi M, Usui H, Katoh-Semba R, Takei K, Goshima Y (2006)
Regulation of dendritic branching and spine maturation by semaphorin3A-Fyn signaling. J Neurosci 26:2971-2980.

Nagel AN, Marshak S, Manitt C, Santos RA, Piercy MA, Mortero SD, Shirkey-Son NJ, Cohen-Cory S (2015) Netrin-1 directs dendritic growth and connectivity of vertebrate central neurons in vivo. Neural Dev 10:14.

Nakamura F, Okada T, Shishikura M, Uetani N, Taniguchi M, Yagi T, Iwakura Y, Ohshima T, Goshima Y, Strittmatter SM (2017) Protein tyrosine phosphatase delta mediates the Sema3A-induced cortical basal dendritic arborization through the activation of Fyn tyrosine kinase. J Neurosci 37:7125-7139.

Nishiyama M, Togashi K, von Schimmelmann MJ, Lim CS, Maeda S, Yamashita N, Goshima Y, Ishii S, Hong K (2011) Semaphorin 3A induces CaV2.3 channel-dependent conversion of axons to dendrites. Nat Cell Biol 13:676-685.

Onesto C, Shutes A, Picard V, Schweighoffer F, Der CJ (2008) Characterization of EHT 1864, a novel small molecule inhibitor of Rac family small GTPases. Methods Enzymol 439:111-129.

Peng SS, Tran TS (2017) Regulation of cortical dendrite morphology and spine organization by secreted Semaphorins: a primary culture approach. Methods Mol Biol 1493:209-222.

Riccomagno MM, Hurtado A, Wang H, Macopson JG, Griner EM, Betz A, Brose N, Kazanietz MG, Kolodkin AL (2012) The RacGAP beta2Chimaerin selectively mediates axonal pruning in the hippocampus. Cell 149:1594-1606.

Roberts AW, Kim C, Zhen L, Lowe JB, Kapur R, Petryniak B, Spaetti A, Pollock JD, Borneo JB, Bradford GB, Atkinson SJ, Dinauer MC, Williams DA (1999) Deficiency of the hematopoietic cell-specific Rho family GTPase Rac2 is characterized by abnormalities in neutrophil function and host defense. Immunity 10:183-196.

Sasaki Y, Cheng C, Uchida Y, Nakajima O, Ohshima T, Yagi T, Taniguchi M, Nakayama T, Kishida R, Kudo Y, Ohno S, Nakamura F, Goshima Y (2002) Fyn and Cdk5 mediate semaphorin-3A signaling, which is involved in regulation of dendrite orientation in cerebral cortex. Neuron 35:907-920.

Schulz J, Franke K, Frick M, Schumacher S (2016) Different roles of the small GTPases Rac1, Cdc42, and RhoG in CALEB/NGC-induced dendritic tree complexity. J Neurochem 139:26-39.

Sharma N, Flaherty K, Lezgiyeva K, Wagner DE, Klein AM, Ginty DD (2020) The emergence of transcriptional identity in somatosensory neurons. Nature 577:392-398.

Shelly M, Cancedda L, Lim BK, Popescu AT, Cheng PL, Gao H, Poo MM (2011) Semaphorin3A regulates neuronal polarization by suppressing axon formation and promoting dendrite growth. Neuron 71:433-446.

Shutes A, Onesto C, Picard V, Leblond B, Schweighoffer F, Der CJ (2007) Specificity and mechanism of action of EHT 1864, a novel small molecule inhibitor of Rac family small GTPases. J Biol Chem 282:35666-35678.

Smith CJ, Watson JD, VanHoven MK, Colon-Ramos DA, Miller DM (2012) Netrin (UNC-6) mediates dendritic self-avoidance. Nat Neurosci 15:731737.

Stoeckli ET (2018) Understanding axon guidance: are we nearly there yet? Development 145:dev151415.

Takegahara N, Kang S, Nojima S, Takamatsu H, Okuno T, Kikutani H, Toyofuku T, Kumanogoh A (2010) Integral roles of a guanine nucleotide exchange factor, FARP2, in osteoclast podosome rearrangements. FASEB J 24:4782-4792.

Tong Y, Chugha P, Hota PK, Alviani RS, Li M, Tempel W, Shen L, Park HW, Buck M (2007) Binding of Rac1, Rnd1, and RhoD to a novel Rho GTPase interaction motif destabilizes dimerization of the plexin-B1 effector domain. J Biol Chem 282:37215-37224.

Toyofuku T, Yoshida J, Sugimoto T, Zhang H, Kumanogoh A, Hori M, Kikutani H (2005) FARP2 triggers signals for Sema3A-mediated axonal repulsion. Nat Neurosci 8:1712-1719.

Tran TS, Kolodkin AL, Bharadwaj R (2007) Semaphorin regulation of cellular morphology. Annu Rev Cell Dev Biol 23:263-292.

Tran TS, Rubio ME, Clem RL, Johnson D, Case L, Tessier-Lavigne M, Huganir RL, Ginty DD, Kolodkin AL (2009) Secreted semaphorins control spine distribution and morphogenesis in the postnatal CNS. Nature 462:1065-1069.

Turner LJ, Nicholls S, Hall A (2004) The activity of the plexin-A1 receptor is regulated by Rac. J Biol Chem 279:33199-33205. 
Waimey KE, Huang PH, Chen M, Cheng HJ (2008) Plexin-A3 and plexin-A4 restrict the migration of sympathetic neurons but not their neural crest precursors. Dev Biol 315:448-458.

Waters JE, Astle MV, Ooms LM, Balamatsias D, Gurung R, Mitchell CA (2008) P-Rex1: a multidomain protein that regulates neurite differentiation. J Cell Sci 121:2892-2903.

Whitford KL, Dijkhuizen P, Polleux F, Ghosh A (2002) Molecular control of cortical dendrite development. Annu Rev Neurosci 25:127149.

Xu H, Xiao T, Chen CH, Li W, Meyer CA, Wu Q, Wu D, Cong L, Zhang F, Liu JS, Brown M, Liu XS (2015) Sequence determinants of improved CRISPR sgRNA design. Genome Res 25:1147-1157.
Yaron A, Huang PH, Cheng HJ, Tessier-Lavigne M (2005) Differential requirement for Plexin-A3 and -A4 in mediating responses of sensory and sympathetic neurons to distinct class 3 Semaphorins. Neuron 45:513-523.

Zanata SM, Hovatta I, Rohm B, Puschel AW (2002) Antagonistic effects of Rnd 1 and RhoD GTPases regulate receptor activity in Semaphorin 3Ainduced cytoskeletal collapse. J Neurosci 22:471-477.

Zhao XF, Kohen R, Parent R, Duan Y, Fisher GL, Korn MJ, Ji L, Wan G, Jin J, Püschel AW, Dolan DF, Parent JM, Corfas G, Murphy GG, Giger RJ (2018) PlexinA2 forward signaling through Rap1 GTPases regulates dentate gyrus development and schizophrenia-like behaviors. Cell Rep 22:456-470. 